\title{
Tracing the evolution of NGC 6397 through the chemical composition of its stellar populations ${ }^{\star, \star \star, \star \star \star}$
}

\author{
K. Lind ${ }^{1,6}$, C. Charbonnel ${ }^{2,3}$, T. Decressin ${ }^{4}$, F. Primas ${ }^{1}$, F. Grundahl ${ }^{5}$, and M. Asplund ${ }^{6}$ \\ ${ }^{1}$ European Southern Observatory (ESO), Karl-Schwarzschild-Strasse 2, 85748 Garching bei München, Germany \\ 2 Geneva Observatory, 51 chemin des Maillettes, 1290 Sauverny, Switzerland \\ ${ }^{3}$ Laboratoire d'Astrophysique de Toulouse-Tarbes, CNRS UMR 5572, Université de Toulouse, 14 Av. E. Belin, 31400 Toulouse, \\ France \\ 4 Argenlader Institut für Astronomie (AIfA), Universität Bonn, Auf dem Hügel 71, 53121 Bonn, Germany \\ 5 Department of Physics \& Astronomy, Århus University, Ny Munkegade, 8000 Århus C, Denmark \\ 6 Max-Planck-Institut für Astrophysik, Karl-Schwarzschild-Strasse 1, 85741 Garching bei München, Germany \\ e-mail: klind@mpa-garching.mpg.de
}

Received 7 July 2010 / Accepted 28 November 2010

\section{ABSTRACT}

\begin{abstract}
Context. The chemical compositions of globular clusters provide important information on the star formation that occurred at very early times in the Galaxy. In particular the abundance patterns of elements with atomic number $z \leq 13$ may shed light on the properties of stars that early on enriched parts of the star-forming gas with the rest-products of hydrogen-burning at high temperatures.

Aims. We analyse and discuss the chemical compositions of a large number of elements in 21 red giant branch stars in the metal-poor globular cluster NGC 6397. We compare the derived abundance patterns with theoretical predictions in the framework of the "wind of fast rotating massive star"'-scenario.

Methods. High-resolution spectra were obtained with the FLAMES/UVES spectrograph on the VLT. We determined non-LTE abundances of $\mathrm{Na}$, and LTE abundances for the remaining 21 elements, including $\mathrm{O}$ (from the [OI] line at $630 \mathrm{~nm}$ ), $\mathrm{Mg}, \mathrm{Al}, \alpha$, iron-peak, and neutron-capture elements, many of which had not been previously analysed for this cluster. We also considered the influence of possible He enrichment in the analysis of stellar spectra.

Results. We find that the Na abundances of evolved, as well as unevolved, stars in NGC 6397 show a distinct bimodality, which is indicative of two stellar populations: one primordial stellar generation of composition similar to field stars, and a second generation that is polluted with material processed during hydrogen-burning, i.e., enriched in $\mathrm{Na}$ and $\mathrm{Al}$ and depleted in $\mathrm{O}$ and $\mathrm{Mg}$. The red giant branch exhibits a similar bimodal distribution in the Strömgren colour index $c_{y}=c_{1}-(b-y)$, implying that there are also large differences in the $\mathrm{N}$ abundance. The two populations have the same composition for all analysed elements heavier than $\mathrm{Al}$, within the measurement uncertainty of the analysis, with the possible exception of [Y/Fe]. Using two stars with almost identical stellar parameters, one from each generation, we estimate the difference in He content, $\Delta Y=0.01 \pm 0.06$, given the assumption that the mass fraction of iron is the same for the stars.

Conclusions. NGC 6397 hosts two stellar populations that have different chemical compositions of N, O, Na, Mg, and probably Al. The cluster is dominated (75\%) by the second generation. We show that massive stars of the first generation can be held responsible for the abundance patterns observed in the second generation long-lived stars of NGC 6397. We estimate that the initial mass of this globular cluster is at least ten times higher than its present-day value.
\end{abstract}

Key words. stars: Population II - globular clusters: individual: NGC 6397 - methods: observational - techniques: spectroscopic stars: abundances

\section{Introduction}

The abundance patterns of light elements (up to $\mathrm{Al}$ ) in globular clusters are in the process of being carefully investigated. In particular, many groups are studying the origin of the larger spread in $\mathrm{C}, \mathrm{N}, \mathrm{O}, \mathrm{Na}, \mathrm{Mg}$, and $\mathrm{Al}$ abundances compared to field stars

* Based on data collected at European Southern Observatory (ESO), Paranal, Chile, under program IDs 077.A-0018(A) and 281.D-5028(A), as well as data collected with the Danish $1.54 \mathrm{~m}$ at European Southern Observatory (ESO), La Silla.

$\star \star$ Tables A.1 and A.2 are only available in electronic form at http: //www. aanda.org

$\star \star \star$ Two tables with line equivalent widths, chemical abundances, and stellar parameters are only available in electronic form at the CDS via anonymous ftp to cdsarc.u-strasbg. fr $(130.79 .128 .5)$ or via http://cdsarc.u-strasbg.fr/viz-bin/qcat?]/A+A/527/A148 of similar metallicity. The present status of the observed light element $(\mathrm{O}, \mathrm{Na}, \mathrm{Mg}$, and $\mathrm{Al})$ abundances in globular clusters and their possible consequences for the formation and enrichment history of these stellar populations have been presented in a series of publications by (Carretta et al. e.g. 2009a, 2010; see also reviews by Gratton et al. 2004; and Charbonnel 2005). The main findings, as inferred from high-resolution spectroscopy of individual globular cluster stars, are apparent enhancements of $\mathrm{N}, \mathrm{Na}$, and $\mathrm{Al}$ abundances and deficiencies in $\mathrm{Li}, \mathrm{O}$, and $\mathrm{Mg}$. These patterns can be naturally explained in terms of the enrichment of the nucleosynthesis rest-products of H-burning at high temperatures (Denisenkov \& Denisenkova 1989; Langer et al. 1993). Since the resulting anti-correlations between the O-Na abundances in particular have not only been seen in evolved red giant branch (RGB) stars, but also in turn-off (TO) and subgiant branch (SGB) stars (Gratton et al. 2001), an intrinsic stellar 
evolutionary cause is very unlikely, i.e. the stars cannot have established these abundance patterns themselves. It is instead believed that the gas that formed second generation stars in globular clusters underwent early pollution by slow ejecta from intermediate or massive stars.

In this context, it is significant that photometric evidence of multiplicity has been found in some clusters, e.g. the parallel main sequences identified in $\omega$ Cen and NGC 2808. These observations seemingly necessitate that a difference in the $\mathrm{He}$ content be present (e.g. Norris 2004), in qualitative agreement with the self-enrichment process responsible for the other lightelement variations. Helium enrichment is also commonly invoked to explain the extended horizontal branch observed in many clusters (D'Antona \& Caloi 2008). We are, however, far from building a fully consistent picture of the chemical evolution of globular clusters that can explain all the various observations simultaneously.

A key unknown is the nature of the polluting objects. One possibility is that so-called hot bottom burning occurs at the base of the convective envelope in intermediate-mass stars during the asymptotic giant branch (AGB) phase, leaving nucleosynthesis products in the envelopes that are subsequently expelled (Ventura \& D'Antona 2008, 2010). In addition, super-AGB stars have been suggested to be responsible for the most extreme anomalies (Pumo et al. 2008). The main alternative scenario is a slow mechanical wind from rapidly rotating massive stars (e.g. Decressin et al. 2007a), whose envelopes have been enriched in H-burning products by means of deep internal mixing (see Sect.4). Yet another option is mass loss from massive binary systems, as suggested by de Mink et al. (2009).

By performing accurate abundance analysis of many elements (and isotopic ratios) in large stellar samples, we may be able to pin-point the nature of the progenitors (e.g. Charbonnel 2005 , and references therein). A common property of the competing scenarios is that the pollution would mainly alter the light element abundances of the second generation stars in globular clusters, thus leaving $\alpha$ and iron-peak elements unaffected. This is necessary to explain the homogeneous composition of these elements seen in most clusters. Elements created in the s-process may be affected by AGB pollution, suggesting that correlations exist between s-process and light element anomalies. In NGC 6752, an unexplained correlation was indeed identified between $\mathrm{Al}$ abundances and $\mathrm{Y}, \mathrm{Zr}$, and $\mathrm{Ba}$ (Yong et al. 2005), but the systematic heavy element variations are small ( 0.1 dex $)$ and comparable to the statistical scatter. One must also bear in mind that the yields of AGB stars are uncertain (e.g. Charbonnel 2007; Decressin et al. 2009; Ventura \& D'Antona 2010).

In addition to mapping the presence of abundance trends and correlations, it is also essential to investigate, preferably with sound number statistics, the fraction of stars with normal chemical compositions, similar to those in the field, and the fraction of second and possibly third generation stars in globular clusters. Linking this information to other cluster observables, one may construct a schematic model for the episodes of star formation and evolution. Carretta et al. (2010) describe a possible general formation scenario in which a precursor population, forming from the gas assembled at a very early epoch inside a CDM halo, efficiently raises the metal content of the gas cloud via corecollapse supernova explosions. These trigger a second, large episode of star formation, the so-called primordial population (first generation). The slow winds of massive or intermediatemass stars of the primordial population feed a cooling flow, and the intermediate (second) generation of stars are formed in the central parts of the cluster, out of material enriched in H-burning products. The remaining gas is dispersed by core-collapse supernovae of the second generation and star formation ceases. The present-day cluster is dominated by the second generation, with a smaller fraction, approximately $30 \%$, being left of the primordial population. Critical factors determining the outcome of this scenario are the initial mass function (IMF) of the polluting stars, the initial total mass of the cluster, and the amount of mixing between processed gas in the slow stellar ejecta and pristine cluster gas. We discuss these issues in Sect. 4.

HST photometry of NGC 6397 produces a remarkably clean HR-diagram, with a very tight main sequence (Richer et al. 2008) and a very compact blue horizontal branch, i.e. there are no obvious photometric signs of multiple populations. The cluster is well-studied in terms of numbers of stars for which highresolution spectra have been obtained, but only a handful of elements have previously been analysed even on the RGB (most recently by Castilho et al. 2000; Gratton et al. 2001; Thévenin et al. 2001; Korn et al. 2007; Carretta et al. 2009a), as summarised in Table 1.

Early studies of the strengths of the $\mathrm{G}$ and $\mathrm{CN}$ band in RGB stars in NGC 6397 (and other clusters) suggested that there are anomalies in their $\mathrm{C}$ and $\mathrm{N}$ abundances (Bell et al. 1979; Briley et al. 1990). Eventually, Gratton et al. (2001) also detected a significant spread in $\mathrm{Na}$ abundance for a sample of ten $\mathrm{TO}$ stars and RGB stars, findings that clearly pointed to an intrinsic, rather than evolutionary, origin. Carretta et al. (2005) corroborated these findings for a larger sample of stars and also found a significant $\mathrm{O}-\mathrm{Na}$ anti-correlation, as well as a large spread in $\mathrm{C}$ and $\mathrm{N}$ abundance (see also Pasquini et al. 2004). In the latest analysis by Carretta et al. (2009b,a), the O-Na anti-correlation is present, although the number statistics are still rather small, oxygen measurements in particular being few in number. $\mathrm{A} \mathrm{Mg}-$ Al anti-correlation has not been identified in NGC 6397, but Mg also seems to exhibit a certain scatter (Korn et al. 2007). Lind et al. (2009), hereafter Paper I, presented $\mathrm{Na}$ abundances for $>100$ TO, SGB, and RGB stars, and found that the most heavily Na-enriched stars are also significantly depleted in Li. It is thus clear that NGC 6397, like other globular clusters, should no longer be regarded as a single stellar population despite the tightness of its colour-magnitude diagram. However, even if pollution indeed seems to have taken place in the cluster, it is unclear to which extent, and which elements are affected by it. In this study, which targets red giants, we cover as many elements as possible for a large sample of stars and give a more decisive answer to this question.

\section{Observations and analysis}

We obtained high-resolution $(R=47000)$ spectra with the multi-object fibre spectrograph FLAMES/UVES on the VLT, covering the wavelength range 480-680 nm (UVES Red 580 standard setting). For a subset of six stars, we also have spectra covering the wavelength range 760-1000 nm (UVES Red 860 standard setting). Our targets are all RGB stars, whose surface abundances should reflect the initial composition of cluster gas, with the notable exceptions of $\mathrm{Li}$ and $\mathrm{Be}$, which are not studied here (Li abundances were published in Paper I). In particular, any internal abundance gradients created by atomic diffusion (Korn et al. 2007; Lind et al. 2008) during the main sequence have been erased by the growth of the convective envelope.

We consider here a subset of the targets analysed in Paper I, and we refer to that study for a detailed description of the observations, data reduction, and stellar parameter determination. In brief, we determined effective temperatures from the 
K. Lind et al.: The chemical evolution of NGC 6397

Table 1. Mean abundances and abundance dispersions compared to literature values for NGC 6397.

\begin{tabular}{|c|c|c|c|c|c|c|c|c|c|c|c|}
\hline & $n_{\text {stars }}$ & $n_{\text {lines }}$ & $\langle A(\mathrm{X})\rangle$ & $\sigma$ & $\mathrm{C} 09^{a, f}$ & $\mathrm{~K} 07^{b, f}$ & ${\mathrm{G} 01^{c}}^{c}$ & ${\mathrm{C} 00^{d}}^{d}$ & $\langle A(\mathrm{X})\rangle_{\mathrm{I}}$ & $\langle A(\mathrm{X})\rangle_{\mathrm{II}}$ & $\Delta A(\mathrm{X})_{\mathrm{II}-\mathrm{I}}$ \\
\hline$[\mathrm{Fe} / \mathrm{H}]$ & 21 & $11-13$ & -2.08 & 0.02 & -1.95 & -2.12 & -2.05 & -2.0 & $-2.05 \pm 0.02$ & $-2.08 \pm 0.01$ & $-0.03 \pm 0.02$ \\
\hline$[\mathrm{O} / \mathrm{Fe}]^{e}$ & 17 & $1-3$ & 0.58 & 0.13 & 0.35 & $\ldots$ & 0.37 & 0.15 & $0.71 \pm 0.04$ & $0.56 \pm 0.03$ & $-0.15 \pm 0.05$ \\
\hline$[\mathrm{Na} / \mathrm{Fe}]$ & 21 & $1-2$ & 0.11 & 0.14 & 0.11 & $\ldots$ & 0.31 & 0.19 & $-0.18 \pm 0.07$ & $0.16 \pm 0.02$ & $0.34 \pm 0.07$ \\
\hline$[\mathrm{Mg} / \mathrm{Fe}]$ & 21 & 1 & 0.31 & 0.07 & 0.25 & 0.37 & 0.20 & $\ldots$ & $0.43 \pm 0.03$ & $0.29 \pm 0.01$ & $-0.14 \pm 0.04$ \\
\hline$[\mathrm{Al} / \mathrm{Fe}]^{e}$ & 21 & $1-2$ & 0.42 & 0.12 & . & $\ldots$ & 0.35 & $\ldots$ & $<0.34$ & $0.44 \pm 0.03$ & $0.10 \pm 0.07$ \\
\hline$[\mathrm{Si} / \mathrm{Fe}]$ & 21 & $2-4$ & 0.24 & 0.07 & 0.28 & $\ldots$ & $\ldots$ & 0.27 & $0.20 \pm 0.02$ & $0.25 \pm 0.02$ & $0.04 \pm 0.03$ \\
\hline$[\mathrm{Ca} / \mathrm{Fe}]$ & 21 & 16 & 0.25 & 0.02 & . & 0.37 & $\ldots$ & 0.20 & $0.25 \pm 0.02$ & $0.25 \pm 0.01$ & $-0.00 \pm 0.02$ \\
\hline$[\mathrm{Sc} / \mathrm{Fe}]$ & 21 & $5-6$ & 0.11 & 0.03 & $\ldots$ & $\ldots$ & $\ldots$ & & $0.10 \pm 0.01$ & $0.12 \pm 0.01$ & $0.01 \pm 0.01$ \\
\hline$[\mathrm{Ti} / \mathrm{Fe}]$ & 21 & $3-5$ & 0.30 & 0.03 & $\ldots$ & 0.22 & $\ldots$ & 0.36 & $0.31 \pm 0.01$ & $0.30 \pm 0.01$ & $-0.02 \pm 0.01$ \\
\hline$[\mathrm{Cr} / \mathrm{Fe}]$ & 21 & $1-2$ & 0.02 & 0.05 & $\ldots$ & $\ldots$ & $\ldots$ & $\ldots$ & $0.03 \pm 0.01$ & $0.02 \pm 0.01$ & $-0.01 \pm 0.02$ \\
\hline$[\mathrm{Mn} / \mathrm{Fe}]$ & 17 & 1 & -0.54 & 0.13 & $\ldots$ & $\ldots$ & $\ldots$ & $\ldots$ & $-0.48 \pm 0.09$ & $-0.55 \pm 0.03$ & $-0.07 \pm 0.09$ \\
\hline$[\mathrm{Co} / \mathrm{Fe}]$ & 13 & 1 & 0.10 & 0.10 & $\ldots$ & $\ldots$ & $\ldots$ & $\ldots$ & $0.12 \pm 0.08$ & $0.09 \pm 0.03$ & $-0.03 \pm 0.09$ \\
\hline$[\mathrm{Ni} / \mathrm{Fe}]$ & 21 & $8-13$ & -0.14 & 0.04 & $\ldots$ & $\ldots$ & $\ldots$ & $\ldots$ & $-0.15 \pm 0.03$ & $-0.14 \pm 0.01$ & $0.01 \pm 0.03$ \\
\hline$[\mathrm{Cu} / \mathrm{Fe}]$ & 13 & 1 & -0.89 & 0.09 & $\ldots$ & $\ldots$ & $\ldots$ & $\ldots$ & $-0.87 \pm 0.05$ & $-0.90 \pm 0.03$ & $-0.03 \pm 0.06$ \\
\hline$[\mathrm{Zn} / \mathrm{Fe}]$ & 21 & 1 & 0.01 & 0.06 & $\ldots$ & $\ldots$ & $\ldots$ & $\cdots$ & $0.02 \pm 0.03$ & $0.01 \pm 0.02$ & $-0.01 \pm 0.03$ \\
\hline$[\mathrm{Y} / \mathrm{Fe}]$ & 21 & 2 & -0.26 & 0.03 & $\ldots$ & $\ldots$ & $\ldots$ & -0.16 & $-0.29 \pm 0.01$ & $-0.25 \pm 0.01$ & $0.04 \pm 0.01$ \\
\hline$[\mathrm{Zr} / \mathrm{Fe}]$ & 5 & 1 & 0.11 & 0.06 & $\ldots$ & $\ldots$ & $\ldots$ & $\ldots$ & $0.14 \pm 0.10$ & $0.10 \pm 0.03$ & $-0.04 \pm 0.11$ \\
\hline$[\mathrm{Ba} / \mathrm{Fe}]$ & 21 & $1-2$ & -0.08 & 0.05 & $\ldots$ & -0.18 & $\ldots$ & -0.16 & $-0.12 \pm 0.04$ & $-0.07 \pm 0.01$ & $0.05 \pm 0.04$ \\
\hline$[\mathrm{Ce} / \mathrm{Fe}]$ & 4 & 1 & 0.42 & 0.04 & $\ldots$ & $\ldots$ & $\ldots$ & $\ldots$ & $0.42 \pm 0.14$ & $0.42 \pm 0.03$ & $0.00 \pm 0.14$ \\
\hline$[\mathrm{Nd} / \mathrm{Fe}]$ & 16 & $3-5$ & 0.18 & 0.05 & 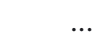 & $\cdots$ & $\ldots$ & $\ldots$ & $0.14 \pm 0.05$ & $0.19 \pm 0.01$ & $0.05 \pm 0.05$ \\
\hline$[\mathrm{Eu} / \mathrm{Fe}]$ & 8 & 1 & 0.40 & 0.06 & $\ldots$ & $\ldots$ & $\ldots$ & $\ldots$ & $0.38 \pm 0.05$ & $0.41 \pm 0.03$ & $0.03 \pm 0.06$ \\
\hline
\end{tabular}

Notes. Listed are also the mean abundances of the RGB stars characterised as belonging to the first generation $\left(\langle A(\mathrm{X})\rangle_{\mathrm{I}}, 3\right.$ stars $)$ and second generation $\left(\langle A(\mathrm{X})\rangle_{\mathrm{II}}, 18\right.$ stars), as well as the difference between the two mean quantities. ${ }^{(a)}$ Carretta et al. $(2009 \mathrm{a})$, mean of 13 targets. [Fe/H] based on FeI lines. ${ }^{(b)}$ Korn et al. (2007), mean of six brightest targets. ${ }^{(c)}$ Gratton et al. (2001), mean of three brightest targets. ${ }^{(d)}$ Castilho et al. (2000), mean of ten brightest targets. ${ }^{(e)}$ Upper limits treated as detections. ${ }^{(f)}$ Asplund et al. (2009) solar abundances adopted.

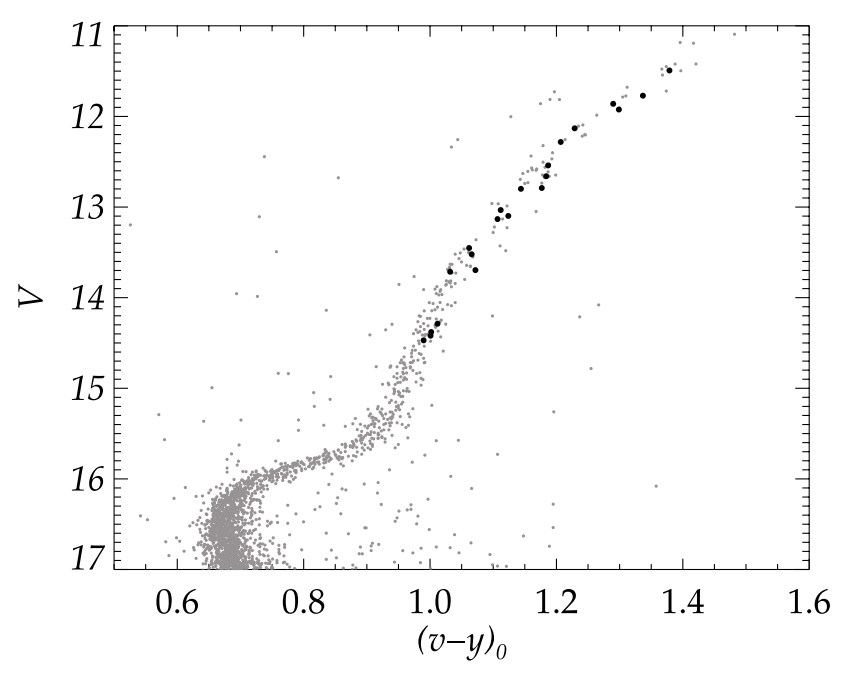

Fig. 1. The targeted RGB stars are marked with black bullets in the $V-$ $(v-y)$ diagram of NGC 6397. The RGB bump is located at $V \approx 12.6$.

$(b-y)$ photometric index, interpolating between data points in the grid of synthetic Strömgren colours computed by Önehag et al. (2009) using MARCS model atmospheres (Gustafsson et al. 2008). Surface gravities were derived from the standard relation to mass and luminosity. In total, we present abundances for 21 red giants distributed along the upper RGB of NGC 6397 (see Fig. 1), above and below the bump. The targets span a total range of approximately $500 \mathrm{~K}$ in effective temperature and 1.5 dex in surface gravity.

The full list of lines used in the analysis is given in Appendix A (except for all iron lines, which are listed in Paper I), with references to the oscillator strengths that were adopted. The wavelengths and the majority of $f$-values were taken from the
Vienna Atomic Line Database (VALD). In all abundance analyses we used 1D, LTE, spherically symmetric, hydrostatic model atmospheres computed with the MARCS code (Gustafsson et al. 2008). For $\mathrm{O}, \mathrm{Na}, \mathrm{Mg}$, and $\mathrm{Al}$ lines, we applied spectrum synthesis with the Uppsala code Bsyn, whereas all other elemental abundances were determined from equivalent width measurements of single lines that were translated to abundances with the corresponding code EQWIDTH. Abundance ratios with respect to the Sun were calculated using the solar abundances of Asplund et al. (2009). The listed abundance errors reflect only the measurement uncertainty, as estimated from the signal-to-noise ratio of the spectra (Norris et al. 2001), and do not consider uncertainties in the adopted stellar parameters. Upper limits to the equivalent width, hence the abundance, were calculated by adding the estimated measurement uncertainty twice to the best-fit synthetic spectrum. Our analysis has its obvious limitations in that it relies mostly on traditional 1D modelling and LTE line formation, and neglects hyperfine splitting. However, the modelling procedure is fully adequate for establishing a robust internal abundance scale, which is our primary objective.

Figure 2 shows the observed and best-fit model spectra of $\mathrm{O}$, $\mathrm{Na}, \mathrm{Mg}$, and Al lines in two RGB stars, \#17691 and \#12138. The abundances of these four elements were all based on weak lines, whose line formation do not depart substantially from LTE. For $\mathrm{Na}$ only, we computed and adopted non-LTE abundances for all targets, whereas LTE was assumed to hold for the other species. The oxygen abundance was determined from the forbidden line at $630 \mathrm{~nm}$, which is believed to have negligible non-LTE corrections, but may be sensitive to granulation effects (Kiselman 2001; Nissen et al. 2002). For the four targets for which this was possible, we also determined the oxygen abundance based on the NIR triplet lines at $777.1 \mathrm{~nm}$ and $777.4 \mathrm{~nm}$, finding very good agreement with the $630 \mathrm{~nm}$ line in LTE. Fabbian et al. (2009) predict non-LTE corrections for the triplet lines to be of the 

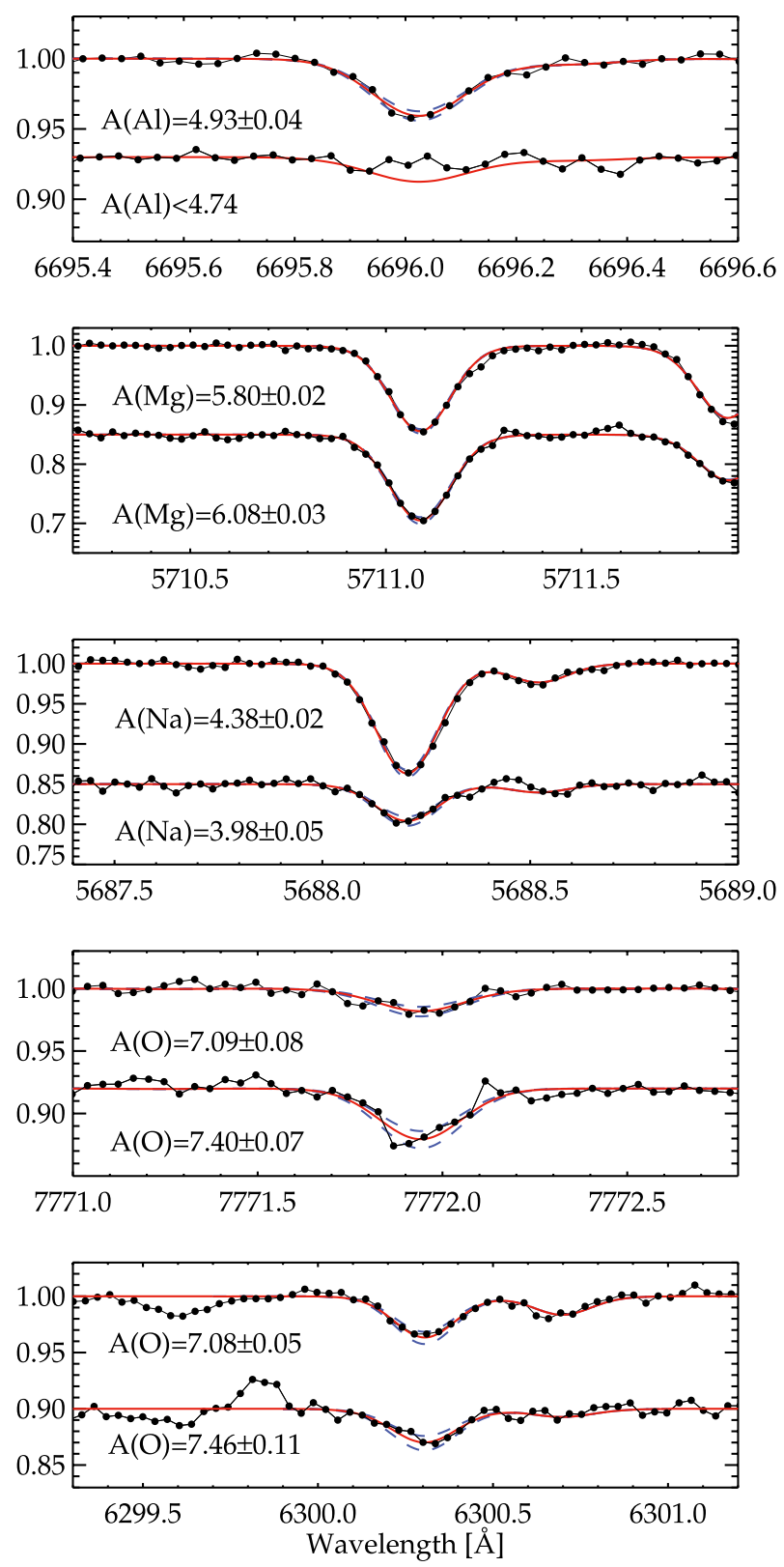

Fig. 2. The figure shows some sample UVES spectra and the best fitting synthetic models and $\pm 1 \sigma$ profiles. The top spectrum in each panel represents a second generation star (12138) and the bottom a first generation star (17691).

order of $-0.05 \ldots-0.08$ dex for our targets, adopting hydrogen collisions with $S_{\mathrm{H}}=1.0$ (Pereira et al. 2009). Applying this correction would slightly lessen the agreement with the forbidden line. However, a different choice of oscillator strength (e.g. that by Storey \& Zeippen 2000), would lower the oxygen abundances inferred from the $630 \mathrm{~nm}$ line by a similar amount.

The $\mathrm{Na}$ abundances were based on the $568.2 \mathrm{~nm}$ and $568.8 \mathrm{~nm}$ doublet lines by interpolating between non-LTE curveof-growths, established with the most recent atomic data for a grid of MARCS model atmospheres (Lind et al. 2011). The nonLTE corrections are moderate, being of the order of $-0.07 \mathrm{dex}$ for stars with these stellar parameters. For five targets, we compared the abundances inferred from the 568.2/568.8 nm lines with those inferred from the $818.3 / 819.4 \mathrm{~nm}$ lines in Paper I. To ensure that the set of $\mathrm{Na}$ abundances are fully consistent with the current study, we used the published equivalent widths in Paper I and recomputed the abundances based on the latest non-LTE calculations. For these near-infra red lines, LTE analysis overestimates the abundance by $0.15-0.35 \mathrm{dex}$, depending on the line strength and stellar parameters. As seen in Fig. 4, the non-LTE corrected abundances agree reassuringly well within the uncertainties.

The $\mathrm{Mg}$ abundance was determined from the $\mathrm{Mg}$ line at $571.1 \mathrm{~nm}$, for which Shimanskaya et al. (2000) report non-LTE corrections that are smaller than 0.02 dex for the relevant stellar parameters. We note that adopting a calculated (e.g. Chang 1990), rather than experimentally determined $f$-value (Lincke \& Ziegenbein 1971) for this line would systematically lower the $\mathrm{Mg}$ abundances by $\sim 0.1 \mathrm{dex}$. The doublet lines of neutral $\mathrm{Al}$ at $669.6 / 669.8 \mathrm{~nm}$ were used to infer the $\mathrm{Al}$ abundance. We did not find any non-LTE calculations in the literature for these lines in metal-poor giant stars, but since they are weak, high-excitation lines, they should at least be more reliable abundance indicators in LTE than the stronger UV resonance lines at 396.1/394.4nm (e.g. Asplund 2005). Furthermore, since our targets all have quite similar stellar parameters, the differential non-LTE effects should be small.

\section{Abundance analysis}

Figure 3 shows $[\mathrm{Fe} / \mathrm{H}]$ and abundance ratios relative to $\mathrm{Fe}^{1}$ for all stars (see individual star abundances in Appendix A). Table 1 lists the mean abundance and dispersion in all elements, compared to values found in the literature. Only recent studies that analysed stars on the upper RGB, in approximately the same magnitude range as our targets, are included in the comparison.

\section{1. $\mathrm{O}, \mathrm{Na}, \mathrm{Mg}$, and $\mathrm{Al}$}

In agreement with previous findings for the cluster (e.g. Carretta et al. 2005, 2009a, and Paper I), we find a large spread in $\mathrm{Na}$ abundance. Figure 4 shows how $\mathrm{Na}$ abundances vary with effective temperature for post-TO stars in the cluster, including the RGB star abundances determined in this study, as well as our improved abundance determinations for the sample of TO, SGB, and RGB stars analysed in Paper I. It is apparent that the mean $\mathrm{Na}$ abundance is higher by approximately $0.2 \mathrm{dex}$ in the RGB stars $\left(T_{\text {eff }}<5600 \mathrm{~K}\right)$, compared to the TO and SGB group $\left(T_{\text {eff }}>5950 \mathrm{~K}\right)$. For these low-mass stars, classical models do not predict any dredge-up of fresh Na produced in situ by proton-capture on pristine ${ }^{22} \mathrm{Ne}$ (see e.g. Charbonnel \& Lagarde 2010), so the reason for the apparent $\mathrm{Na}$ enhancement must lie elsewhere. A similar difference was identified by D'Orazi et al. (2010), between dwarfs and giants in 47 Tuc, which they propose to be an artificial result, stemming from the assumption of Gaussian line profiles when determining the equivalent widths of the $818.3 / 819.4 \mathrm{~nm}$ lines. This is not the case for our study, since we use a full spectrum synthesis. Considering in particular the good agreement between the $818.3 / 819.4 \mathrm{~nm}$ lines and the $568.2 / 568.8 \mathrm{~nm}$ lines for the five RGB stars for which both are observed (the mean difference between the doublet is $0.03 \pm 0.04$, we propose that the 0.2 dex difference is real. This is indeed the amount expected from stellar structure models including atomic diffusion, moderated by a certain degree of turbulence below the outer convection zone, as illustrated in Fig. 4 (see Richard et al. 2005, and references therein). Korn et al. (2007) and Lind et al. (2008) found differences of similar size between dwarf and giant star abundances of Fe and Mg in NGC 6397, that are well reproduced by the T6.0 model of Richard et al. We therefore propose

$$
{ }^{1}[X / Y]=\log \left(\frac{N(X)}{N(Y)}\right)_{*}-\log \left(\frac{N(X)}{N(Y)}\right)_{\odot} .
$$


K. Lind et al.: The chemical evolution of NGC 6397
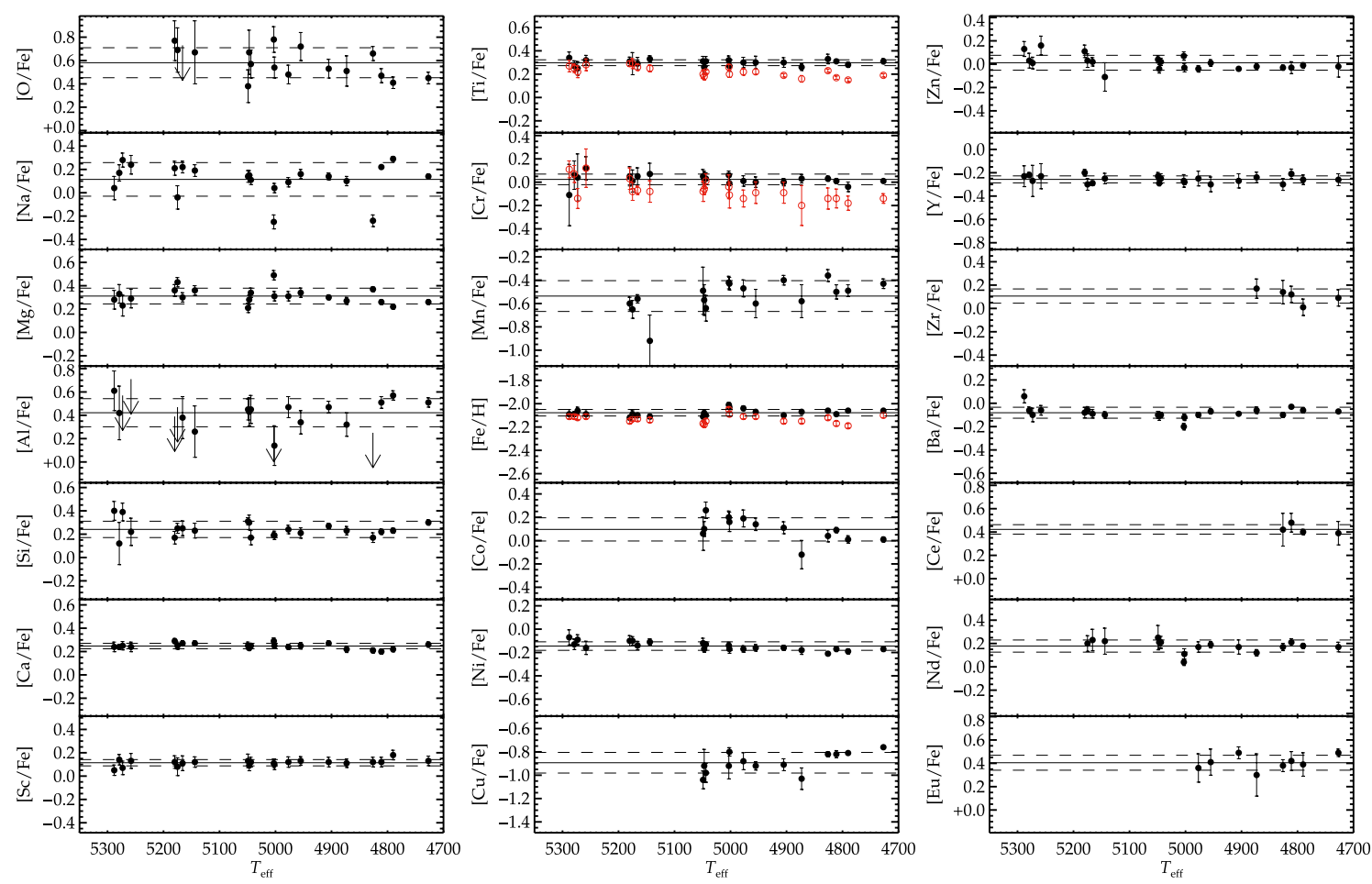

Fig. 3. Elemental abundances of all targets. Each panel spans 1 dex in abundance and marks the average abundance (solid line) and the $1 \sigma$ dispersion limits (dashed lines). Two different symbols are used for $[\mathrm{Ti} / \mathrm{Fe}],[\mathrm{Cr} / \mathrm{Fe}]$, and $[\mathrm{Fe} / \mathrm{H}]$ : the red open circles and the black bullets in these panels represent abundances inferred from neutral and singly ionised lines, respectively. The hottest star in each panel marks the completeness limit, i.e. all sample stars cooler than this limit are shown.

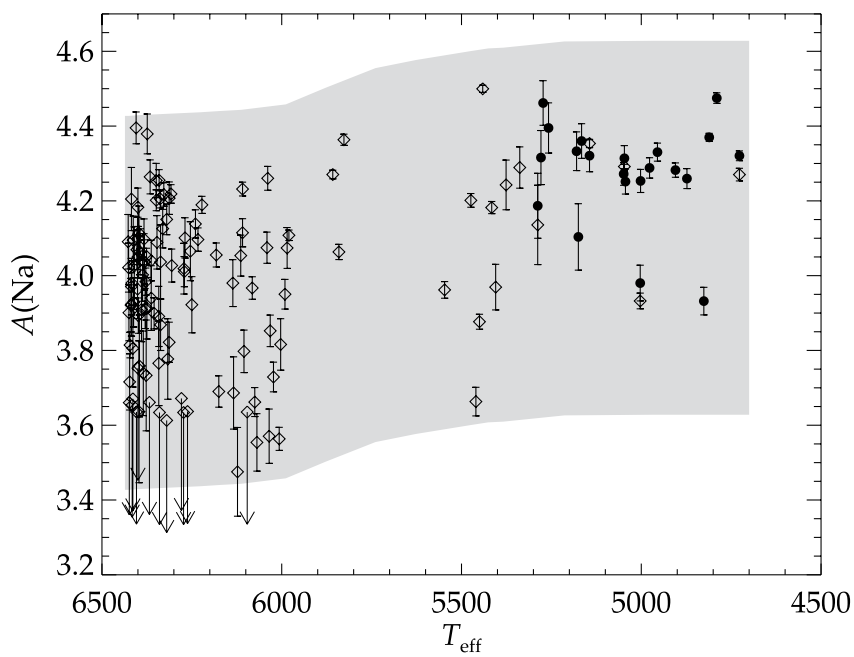

Fig. 4. Non-LTE Na abundance variation with effective temperature in NGC 6397. Bullets represent the RGB stars that are analysed in this study (abundance based on 568.2/568.8 $\mathrm{nm}$ lines) and open diamonds the stars analysed in Paper I (Lind et al. 2009, abundance based on $818.3 / 819.4 \mathrm{~nm}$ lines). The studies have five targets in common. The grey shaded area illustrates, given a range of different initial $\mathrm{Na}$ abundances, the predicted evolution of the surface abundance based on a model with atomic diffusion and turbulence below the outer convective zone (Richard et al. 2005, model T6.0).

that the TO and SGB stars have lower photospheric Na levels because of gravitational settling from the envelope, whereas the initial abundance has been reinstated in the photospheres of RGB stars, by the dredge-up of segregated pristine material when the convection zone deepened. We note that even if atomic diffusion accounts accurately for the abundance differences between dwarfs and giants in NGC 6397, the situation may be different for 47 Tuc. This cluster has a higher metallicity $([\mathrm{Fe} / \mathrm{H}]=-0.76$, Koch \& McWilliam 2008) and hosts TO stars with cooler effective temperatures and larger convective envelopes, which implies that settling is less efficient in this cluster.

Accounting for the presumed effect of atomic diffusion and adjusting the Na abundances of TO and SGB stars by +0.2 dex, the abundance histograms shown in Fig. 5 reveal an interesting pattern. The Na abundance distribution of both groups clearly appears to be bimodal, having two peaks separated by $\approx 0.4 \mathrm{dex}$ and the one at higher abundance being larger than the other. The right hand panel of Fig. 5 shows the histogram of the merged sample, where the two peaks have become more distinguished. A two-population fit, assuming Gaussian distributions, is also indicated by a red solid line. The best fit places the first population around $A(\mathrm{Na})=3.84 \pm 0.10$ (mean and standard deviation) or correspondingly $[\mathrm{Na} / \mathrm{Fe}] \approx-0.30$, and the second around $A(\mathrm{Na})=4.23 \pm 0.13$ or $[\mathrm{Na} / \mathrm{Fe}] \approx 0.09$. The number ratio is approximately $1: 3$, with $25 \%$ of the stars in the first group and $75 \%$ in the second group. We interpret this abundance distribution as a result of intra-cluster pollution, raising the $\mathrm{Na}$ abundances of a second generation of stars relative to the first. This clear distinction between the present populations was impossible before for NGC 6397, probably because of small number statistics. A one-sided Anderson-Darling test of the cumulative distribution function returns a probability of $11 \%$ that the $\mathrm{Na}$ abundances can be described by a single normal distribution with the mean and standard deviation of the full sample $(\langle A(\mathrm{Na})\rangle=4.17$ and $\sigma=0.21$ ).

Two of the RGB stars analysed in this study have Na abundances that clearly place them in the first generation $(A(\mathrm{Na})<$ 4.0, \#5644 and \#17691). One additional star may be regarded as a limiting case $(A(\mathrm{Na})=4.10 \pm 0.09, \# 14592)$, but after 


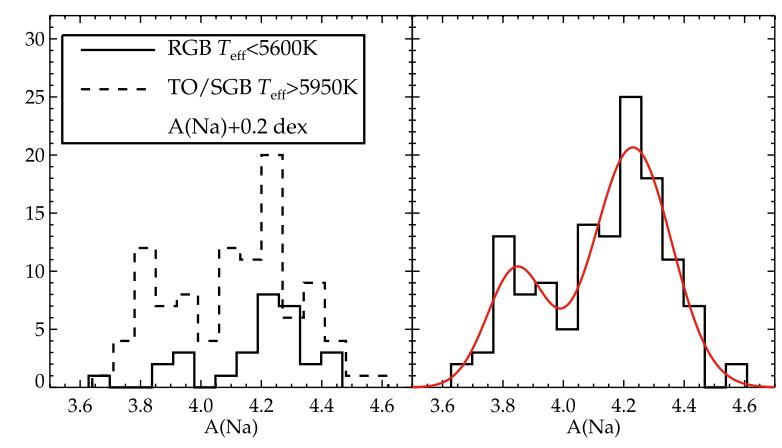

Fig. 5. The left hand panel shows two histograms of the abundances displayed in Fig. 4, separating RGB stars (solid line) from TO and SGB stars (dashed line). A shift of +0.2 dex has been added to the TO/SGB group to correct for the presumed effects of atomic diffusion (see text). The right hand panel shows a histogram of the whole sample and a double-Gaussian fit to the distribution.

considering its comparably high $\mathrm{Mg}$ and $\mathrm{O}$ levels we also assign this star to the first generation. The remaining $18 \mathrm{RGB}$ stars are assigned to the second generation. We ascribe to low number statistics the finding that only three out of 21 RGB stars belong to the first generation (since the number ratio found for the full sample is approximately 1:3, we would have expected five stars). As can be seen from Fig. 6, displaying the $\mathrm{O}, \mathrm{Na}, \mathrm{Mg}$, and $\mathrm{Al}$ abundances, the populations are most clearly distinguished in a $\mathrm{Na}-\mathrm{Mg}$ diagram, probably because of the more precise determination of these abundance ratios compared to $\mathrm{O}$ and $\mathrm{Al}$. The $\mathrm{Al}$ lines are not detectable in the first generation stars and we can only infer upper limits to their $\mathrm{Al}$ abundance. Nevertheless, the abundances of $\mathrm{O}, \mathrm{Al}$, and $\mathrm{Mg}$ support the view that the $\mathrm{Na}$ rich stars have been polluted by gas enriched in H-burning products. Table 1 lists the mean abundance ratios relative to iron of the stars that we characterise as belonging to the first and second generation. The difference in mean abundance ratios is also listed.

Carretta et al. (2009b,a) measured the Na abundances of a large number of RGB stars in NGC 6397 and reported O abundances or upper limits for a subset of 13 stars. Table 1 lists the mean $[\mathrm{O} / \mathrm{Fe}],[\mathrm{Na} / \mathrm{Fe}]$, and $[\mathrm{Mg} / \mathrm{Fe}]$ ratios for this subset, using the same solar abundances as in this study (Asplund et al. 2009). Accounting for the $\sim 0.14$ dex difference in mean $[\mathrm{Fe} / \mathrm{H}]$ between the studies, the mean $\mathrm{O}, \mathrm{Na}$, and $\mathrm{Mg}$ abundances agree rather well $( \pm 0.1$ dex $)$. However, the full set of $\mathrm{Na}$ abundances published by Carretta et al. (including the lower resolution measurements with VLT/GIRAFFE) shows a systematic offset towards higher values by typically 0.2 dex. The reason for this offset can be traced to differences in the adopted oscillator strengths ( $\sim 0.05 \mathrm{dex})$, as well as differences in the non-LTE corrections applied $(\sim 0.15 \mathrm{dex})$.

According to the distinction recognised by Carretta et al. the primordial (first) generation consists of stars with $[\mathrm{Na} / \mathrm{Fe}] \leq$ $[\mathrm{Na} / \mathrm{Fe}]_{\min }+0.3 \mathrm{dex}$, where $[\mathrm{Na} / \mathrm{Fe}]_{\min }$ is the minimum value observed in the cluster and 0.3 dex corresponds to $\sim 4 \sigma[\mathrm{Na} / \mathrm{Fe}]$ and $\sigma[\mathrm{Na} / \mathrm{Fe}]$ is the star-to-star error in $[\mathrm{Na} / \mathrm{Fe}]$. Adopting this definition for our RGB sample, the first generation would consist of the same three stars, $\left([\mathrm{Na} / \mathrm{Fe}]_{\min }=-0.25\right)$, but barely overlap to also include two of our second-generation targets. All the other sample stars would be considered as second-generation stars. Furthermore, Carretta et al. define an extreme component among the second generation stars, if present, as stars having $[\mathrm{O} / \mathrm{Na}]<-0.9$ (or $[\mathrm{O} / \mathrm{Na}]<-1.03$, adopting the solar abundances of Asplund et al. 2009). For NGC 6397, none of the

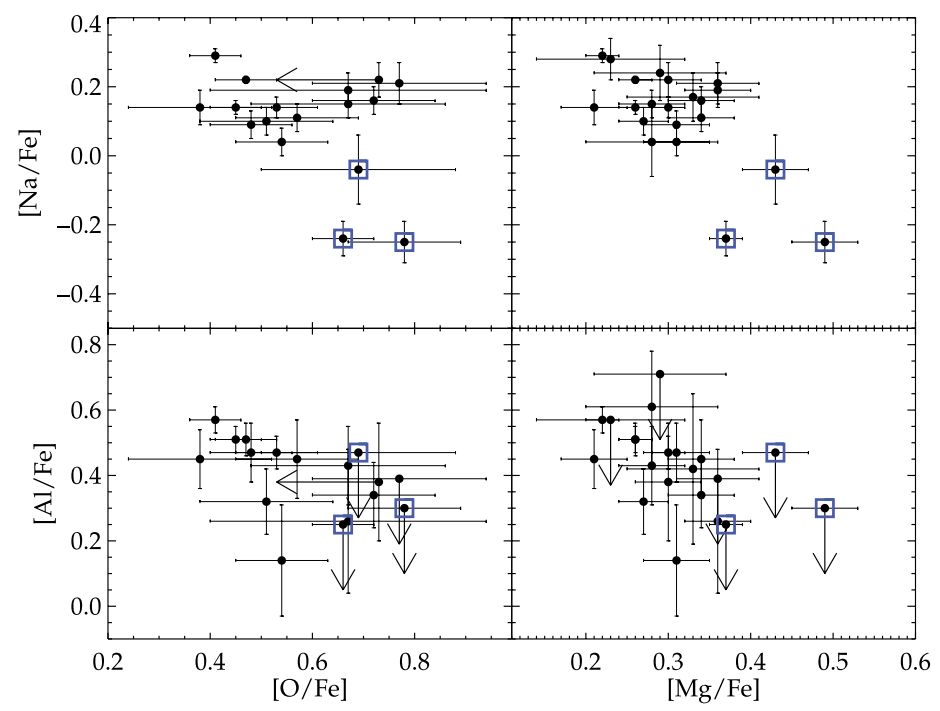

Fig. 6. $\mathrm{Na}, \mathrm{Mg}$, and $\mathrm{Al}$ abundances for 21 stars and $\mathrm{O}$ abundances for 17 stars. The three Na-poor stars that we assign to the first generation are marked with by blue squares.

13 targets analysed by Carretta et al. fall in the extreme component according to this definition and the same is true for our sample. In numbers, Carretta et al. find that $25 \pm 13 \%$ and $75 \pm 22 \%$ of their full sample of stars in NGC 6397 belong to the primordial and intermediate components, respectively, which agrees perfectly with our results (25\% and $75 \%)$.

Finally, we have also inspected the $\mathrm{Na}$ abundance histogram of the full Carretta et al. (2009) sample, to see whether the same double-peaked distribution as in Fig. 5 can be distinguished. This is not the case however, which we believe is likely due to the larger measurement errors (typically $\sim 0.1 \mathrm{dex}$, according to their estimate, compared to $\sim 0.05 \mathrm{dex}$ in our study) stemming from their lower spectral quality. The possibility that the bimodality seen in our abundance data is artificial and not representative of the true distribution obviously cannot be completely ruled out. This is unlikely though, especially considering that the two samples (TO+SGB and RGB) display a very similar pattern. Furthermore, our Na abundance histogram for NGC 6397 bears a striking resemblance to the one found by Marino et al. (2008, see their Fig. 6) for the higher metallicity cluster M4. On the basis of this histogram, the authors find that $\sim 36 \%$ of the stars are centred around $[\mathrm{Na} / \mathrm{Fe}]=0.07 \pm 0.01$, whereas the remaining majority of stars are of second generation, centred around $[\mathrm{Na} / \mathrm{Fe}]=0.38 \pm 0.01$.

Figure 7 compares the abundance ratios of our cluster targets to halo field data. As expected, we see that the $[\mathrm{Na} / \mathrm{Fe}]$ ratio of the first generation is in good agreement with the field at this metallicity, whereas the $\mathrm{Na}$ abundances of the second generation are clearly enhanced. The $[\mathrm{Al} / \mathrm{Fe}]$ ratios of the second generation also appear enhanced relative to the field, but only upper limits can be inferred for the first generation, limiting our comparison to field stars. The $[\mathrm{Mg} / \mathrm{Fe}]$ and $[\mathrm{O} / \mathrm{Fe}]$ ratios have smaller total ranges in the cluster than $[\mathrm{Na} / \mathrm{Fe}]$ and $[\mathrm{Al} / \mathrm{Fe}]$, which reflects that the absolute abundances of $\mathrm{Mg}$ and $\mathrm{O}$ are higher than those of $\mathrm{Al}$ and $\mathrm{Na}$ by almost three orders of magnitude. The measurements of $\mathrm{O}$ and $\mathrm{Mg}$ for both first and second generation stars may be compatible with those of the field. 


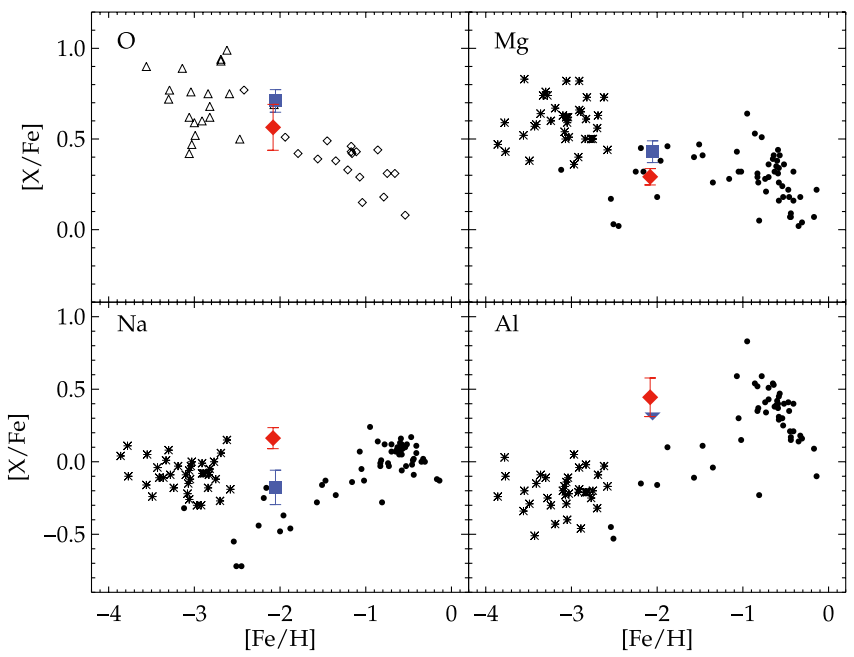

Fig. 7. Comparison between abundances of RGB stars in NGC 6397 and halo field stars. The blue filled squares represent the mean abundances of the NGC 6397 stars assigned to the first generation and the red diamonds the mean of the second generation. The error bars mark the $1 \sigma$ dispersion. Upper limits are treated as detections for the second generation averages for $\mathrm{Al}$ (two stars) and $\mathrm{O}$ (one star) when computing the mean and standard deviation. The literature data for halo field stars are assembled as follows; bullets represent Gehren et al. (2006), star symbols represent Andrievsky et al. (2007, 2008, 2010), open triangles Cayrel et al. (2004), and open diamonds Nissen et al. (2002).

\subsection{Nitrogen}

As mentioned in the introduction, NGC 6397 photometric data appear to be very homogeneous. However, colour indices designed specifically to trace elemental abundances may provide further support for the presence of multiple populations. As realised by Grundahl et al. (2002), the strength of the Strömgren $c_{1}$-index can be used as a tracer of the $\mathrm{N}$ abundance, since the $u$ filter involved in constructing this index covers the $336 \mathrm{~nm} \mathrm{NH}$ features. Yong et al. (2008a) empirically defined a more suitable index for the purpose, $c_{y}=c_{1}-(b-y)$, which removes much of the temperature sensitivity of $c_{1}$. The top panel of Fig. 8 shows a $V-c_{y}$ diagram, in which we have marked the first and second generation targets in this study with different symbols. We have also added the RGB targets analysed in Paper I, assigning stars with $A(\mathrm{Na})<4.0$ to the first generation and $A(\mathrm{Na})>4.0$ to the second. The clear separation between the generations visible in Fig. 8 indicates that their $\mathrm{N}$ abundances are also affected. This correlation between $c_{y}$-index and light element abundances was also illustrated by Milone et al. (2010) for the more metalrich globular cluster NGC 6752. In both clusters, the first generation of stars seem to populate a tight, blue RGB sequence in this colour index, and the second generation a more dispersed, red sequence. We also note the close analogy with the divided Na-rich and Na-poor RGB sequences in the $U-(U-B)$ diagram of M4, as shown by Marino et al. (2008).

In the bottom panel of Fig. 8, we have extracted a box centred on the middle RGB and produced a histogram of the $c_{y}$ index. The magnitude cuts are applied to reduce any remaining bias due to temperature effects. We again see a two-peaked histogram, very similar to the one obtained for the Na abundance. The best fit returns the peak positions at $c_{y}=-0.341$ and $c_{y}=-0.306$, with a size relationship of $25 \%$ and $75 \%$ for the left and right peak, in excellent agreement with previously inferred number fractions for the first and second stellar generation (see Sect. 3.1). However, the probability that the colours can be
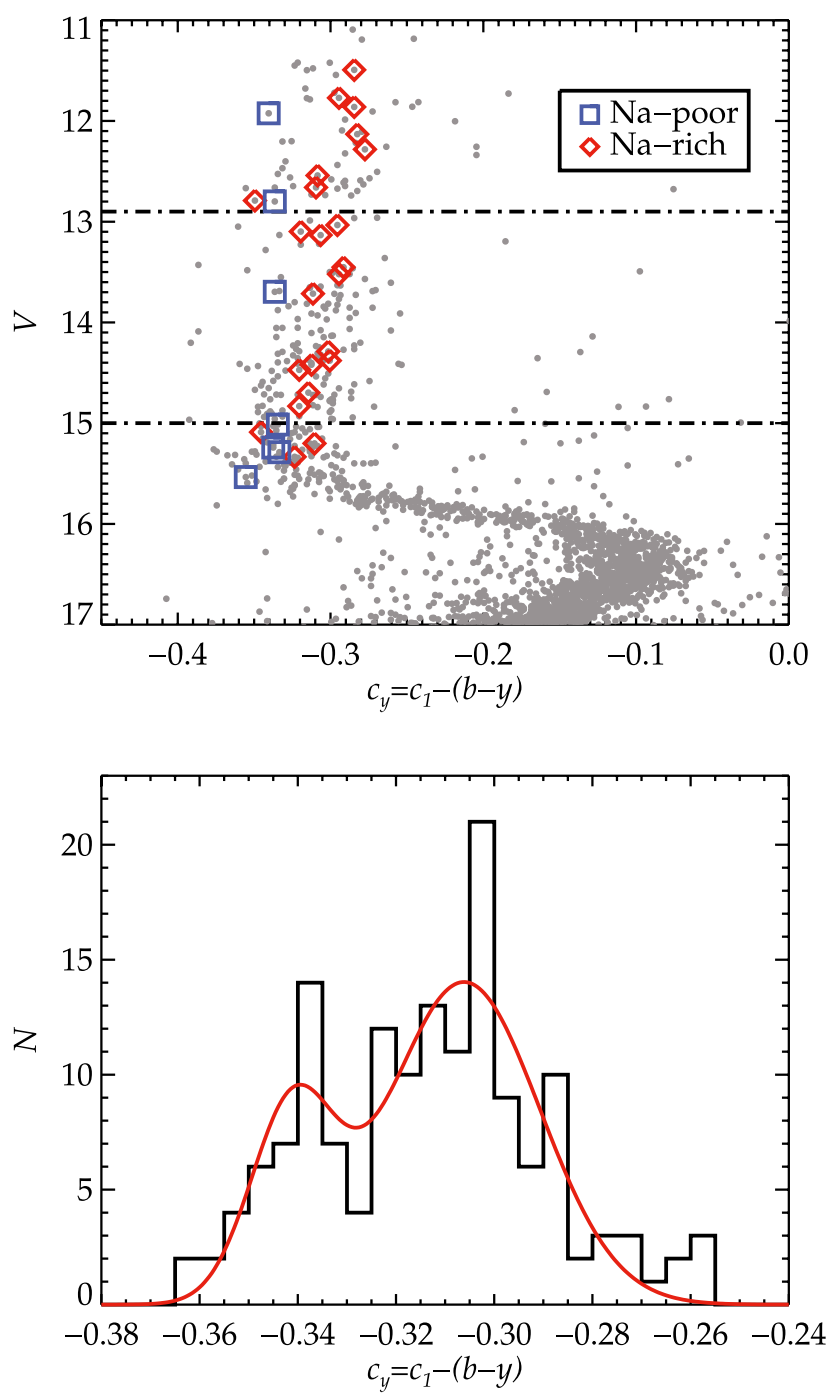

Fig. 8. The top panel shows the $V-c_{y}$ diagram of NGC 6397. Na-poor and Na-rich stars are indicated by blue squares and red diamonds respectively (abundance data from this study and Paper I). The bottom panel shows a histogram of the $c_{y}$ index for red giants in the magnitude range $12.9<V<15$. For details, we refer to the main text.

described by a single Gaussian distribution is $59 \%$, again as given by the Anderson-Darling statistics.

We can estimate the corresponding difference in $[\mathrm{N} / \mathrm{Fe}]$ abundance using the theoretical calculations (based on synthetic colours) by Önehag et al. (2009), giving an approximate sensitivity of $\delta c_{y} / \delta[\mathrm{N} / \mathrm{Fe}]=0.04$ for stars in the same $T_{\text {eff }}$ and $\log g$-ranges in NGC 6752. A somewhat stronger sensitivity, 0.06, was found by Yong et al. (2008a) who empirically correlated spectroscopic $\mathrm{N}$ abundances with $c_{y}$-colour for the same stars. This cluster has a slightly higher metallicity, but still helps us to obtain an order of magnitude estimate. The typical $\Delta[\mathrm{N} / \mathrm{Fe}]$ between stars of the first and second generation thus lies in the range $1-1.5$ dex. This is consistent with spectroscopic $\mathrm{N}$ measurements by Carretta et al. (2005), who found that six out of nine SGB stars are strongly enhanced in $[\mathrm{N} / \mathrm{Fe}]$ compared to the remaining sample, by as much as 1.5 dex. Pasquini et al. (2008) find all three stars in their sample to be highly N-enriched relative to the field. Spectroscopic N measurements for a large sample of stars in NGC 6397 is obviously desirable to establish the absolute range of abundances and to verify the apparent bimodality. 


\section{3. $\alpha$ and iron-peak elements}

Globular clusters are generally very homogeneous in $\alpha$ and ironpeak elements, with the exceptions of peculiar clusters such as $\omega$ Cen. In NGC 6397, the star-to-star scatter in $[\mathrm{Fe} / \mathrm{H}]$ in our RGB sample is extremely small, only $0.03 \mathrm{dex}$, when inferring the iron abundance from singly ionised lines. The Fe I line abundances exhibit a slight decreasing trend with decreasing effective temperature, which is not seen in Fe II line abundances. The same pattern is seen for $[\mathrm{Ti} / \mathrm{Fe}]$ and $[\mathrm{Cr} / \mathrm{Fe}]$ ratios, and may indicate that the temperature scale contains a small systematic bias $(\sim 50 \mathrm{~K}$ over a total range of $500 \mathrm{~K})$ or that non-LTE and/or 3D effects have minor differential effects. In Table 1, the reported $\mathrm{Fe}, \mathrm{Ti}$, and $\mathrm{Cr}$ abundances are all based entirely on singly ionised lines. The abundances of $\mathrm{Mn}, \mathrm{Co}, \mathrm{Cu}$, and $\mathrm{Zn}$ are based on one rather weak neutral line, which likely explains the larger star-tostar scatter in these abundances.

In general, the $\alpha$ and iron-peak elements display the expected pattern, in agreement with the halo field at this metallicity. The abundances of $\mathrm{Ca}, \mathrm{Si}, \mathrm{Ti}$, and $\mathrm{Sc}$ are overabundant by $0.1-0.3 \mathrm{dex}$, whereas the iron-peak elements $\mathrm{Cr}, \mathrm{Co}, \mathrm{Ni}$, and $\mathrm{Zn}$ are approximately solar. The odd-z elements $\mathrm{Mn}$ and $\mathrm{Cu}$ are strongly under-abundant relative to the Sun, and the low value we inferred for $\mathrm{Cu}(\langle[\mathrm{Cu} / \mathrm{Fe}]\rangle=-0.89)$ is slightly below the values found for field dwarfs at this metallicity by Primas \& Sobeck (2008). The mean $\mathrm{Mn}$ abundance $(\langle[\mathrm{Mn} / \mathrm{Fe}]\rangle=-0.54)$ is very similar to the LTE abundances inferred by Bergemann \& Gehren (2008), but as shown in that study, non-LTE corrections for Mn are positive and rather substantial.

As can also be seen in Table 1, there is no significant difference (i.e. $>2 \sigma$ ) between the mean abundances of intermediate mass (heavier than $\mathrm{Al}$ ) and iron-peak elements for the stars that we characterise as belonging to the first and second generation.

\subsection{Neutron capture elements}

Metal-poor halo field stars are known to display a large scatter in some of the neutron capture elements, which cannot be explained by observational uncertainties (see e.g. Barklem et al. 2005; Burris et al. 2000). Stars within a globular cluster are generally far more homogeneous in their heavy element abundances, as shown by for example James et al. (2004) for NGC 6397, NGC 6752, and 47 Tuc and by Yong et al. (2008b) for M4 and M5. This is confirmed by our study, in which small abundance dispersions are found for $\mathrm{Y}, \mathrm{Zr}, \mathrm{Ba}, \mathrm{Ce}, \mathrm{Nd}$, and $\mathrm{Eu}$. The mean abundance ratios we derive for $[\mathrm{Y} / \mathrm{Fe}]=-0.26$ and $[\mathrm{Ba} / \mathrm{Fe}]=-0.08$ are in reasonable agreement with previous estimates for the cluster (in addition to the values listed in Table 1, James et al. determined $[\mathrm{Y} / \mathrm{Fe}]=-0.2$ and $[\mathrm{Ba} / \mathrm{Fe}]=-0.17$ for stars at the base of the RGB). The other elements have not been previously analysed in NGC 6397. By comparing to the values determined for the higher metallicity cluster NGC 6752 by Yong et al. (2005), we can observe good agreement between $\mathrm{Zr}$, $\mathrm{Ba}, \mathrm{Ce}, \mathrm{Nd}$, and $\mathrm{Eu}$ abundances. However, for $\mathrm{Zr}$ this is partly coincidental; accounting for differences in the adopted oscillator strength for the $\mathrm{Zr}$ II line at $511.2 \mathrm{~nm}$, our abundance ratio would be 0.3 dex lower. A similar difference is seen when comparing the estimates for $[\mathrm{Y} / \mathrm{Fe}]$. It thus seems that the two clusters are very similar in heavy neutron-capture elements having $Z>56$, whereas the lighter $Z=39-40$ elements are 0.3 dex less abundant in NGC 6397.

According to Burris et al. (2000), Y, Zr, Ba, and Ce are all mainly s-process elements (72-85\% $s$-process contribution), whereas $\mathrm{Nd}$ is approximately half $r$-process, half s-process. Eu is an almost pure (97\%) $r$-process element. For the first time, we determine the $[\mathrm{Ba} / \mathrm{Eu}]$ ratio in this cluster, and the value we infer $(-0.49)$ is quite close to the scaled solar $r$-process of -0.81 (the scaled s-process value is 1.45 ). The same is true for the $[\mathrm{Zr} / \mathrm{Eu}],[\mathrm{Ce} / \mathrm{Eu}]$, and $[\mathrm{Nd} / \mathrm{Eu}]$ ratios, whereas $[\mathrm{Y} / \mathrm{Fe}]$ is even slightly below the value expected for a pure $r$-process contribution. This implies that chemical enrichment by core-collapse supernovae may be sufficient to explain the observed heavy element abundances, and that the contribution from AGB stars has been limited.

The two stellar generations are very similar in terms of the measured neutron-capture elements (see Table 1). Only the $[\mathrm{Y} / \mathrm{Fe}]$ ratio is higher by $\sim 3 \sigma$ in the second generation. The difference $(0.04 \mathrm{dex})$ is, however, too small to be convincing. A comparison with halo field stars does not provide much information, since the stars in the field are highly scattered. The abundances we find for NGC 6397 are well within the ranges found e.g. by François et al. (2007) for a sample of metal-poor field giants.

\subsection{Helium}

If the second generation stars in globular clusters have indeed been enriched in nucleosynthesis products connected to hydrogen-burning, they should also have a higher He content than their first generation counterparts, although we may only speculate about the exact amount.

di Criscienzo et al. (2010) used the accurate, proper-motion cleaned HST photometry of Richer et al. (2008) to measure the width of the main sequence in NGC 6397 and constrain the possible helium spread in the cluster. Under the assumption that $70 \%$ of the stars belong to the second generation and have a $\mathrm{C}+\mathrm{N}+\mathrm{O}$ enhancement as suggested by spectroscopic observations $(\Delta[(\mathrm{C}+\mathrm{N}+\mathrm{O}) / \mathrm{Fe}] \sim 0.25$ according to Carretta et al. 2005), they conclude that the cluster must be very uniform in $\mathrm{He}$ abundance, implying that there is a difference of only $\Delta Y=0.02$ between the first and second generations. When allowing for higher $[(\mathrm{C}+\mathrm{N}+\mathrm{O}) / \mathrm{Fe}]$ overabundances, a broader spread in helium ( $\Delta Y$ of up to 0.04 ) is compatible with the tightness of the main sequence. Here we attempt to constrain this difference using spectroscopic arguments instead.

The He abundance cannot be measured directly in late-type stars, but nevertheless influences the emerging stellar spectrum. The effects of helium-enrichment in a cool stellar atmosphere has been investigated by Strömgren et al. (1982), who found that changes in the helium-to-hydrogen ratio of $\mathrm{F}$ type dwarfs affect the mean molecular weight of the gas and have an impact on the gas pressure. However, helium contributes only negligible to the line and continuous opacity in a cool atmosphere and does not provide free electrons. Therefore, Strömgren et al. concluded and demonstrated that a helium-enrichment can be mapped merely as a shift in surface gravity, i.e. a heliumenriched atmosphere is similar to a helium-normal atmosphere with a higher surface gravity, in terms of temperature structure and electron pressure structure. Stars with identical $T$ and $P_{\mathrm{e}}$ structures and the same ratio of metals-to-hydrogen also have identical spectra. The calculation of the necessary shift in surface gravity is given by the relation (see equivalent Eq. (12) in Strömgren et al. 1982)

$$
\log g^{\prime \prime}=\log g^{\prime}+\log \left(\frac{\left(1+4 \times y^{\prime}\right)\left(1+y^{\prime \prime}\right)}{\left(1+4 \times y^{\prime \prime}\right)\left(1+y^{\prime}\right)}\right)
$$

where $\log g^{\prime}$ is the surface gravity of the model with heliumto-hydrogen ratio $y^{\prime}=N_{\mathrm{He}} / N_{\mathrm{H}}$ and analogously for the 
Table 2. Stellar parameters for \#5644 and \#14 565 derived assuming a normal-star hydrogen and helium composition.

\begin{tabular}{|c|c|c|c|c|c|}
\hline ID & $\begin{array}{c}T_{\mathrm{eff}} \\
b-y\end{array}$ & $\begin{array}{l}T_{\text {eff }} \\
\mathrm{H} \alpha\end{array}$ & $\begin{array}{c}T_{\text {eff }} \\
\text { Ex. eq. }\end{array}$ & $\begin{array}{c}\log g \\
\text { Photo. }\end{array}$ & $\begin{array}{c}\log g \\
\text { Ion. eq }\end{array}$ \\
\hline 5644 & 4826 & 4740 & 4597 & 1.74 & 1.50 \\
\hline 14565 & 4811 & 4730 & 4598 & 1.71 & 1.52 \\
\hline$\Delta$ & 15 & 10 & -1 & 0.03 & -0.02 \\
\hline
\end{tabular}

double-primed variables. We tested whether the relation also holds for our RGB targets by computing two metal-poor MARCS atmospheric models (Asplund et al. 1997) with $T_{\text {eff }}=$ $4700 \mathrm{~K}$. One model has extreme helium-enrichment, $\log g=$ 1.65 , and $y=1.0$ (corresponding to helium mass-fraction of $Y=0.8$ ). The second model is helium-normal, but has a higher surface gravity, $\log g=1.95$, and $y=0.085(Y=0.25)$. All metal abundances relative to hydrogen are the same in both models $([\mathrm{Fe} / \mathrm{H}]=-2.0)$, which means that the helium-rich model has a lower mass fraction of metals. The temperature structure and electron density structure of the two model atmospheres are very similar, as expected, and the derived elemental abundances based on the models are the same to within $\sim 0.005$ dex. We thus consider it safe to use the scaling with surface gravity of normal composition models to mimic helium-enhancement.

We selected one star from each generation to test the hypothesis of a difference in He-content. The stars have essentially identical stellar parameters, which allows a differential comparison. Table 2 lists the derived stellar parameters for these two targets. The absolute $T_{\text {eff-values inferred from fitting the wings }}$ of the $\mathrm{H} \alpha$ line and from the excitation equilibrium of neutral iron are both somewhat lower than the values derived from the photometric data, but the difference between the two selected stars is small for all methods. Since the true difference in He abundance between the stars, if any, is unknown, the effective temperatures have been computed by assuming a normal heliumto-hydrogen ratio in both objects. None of the methods should be severely biased however by this assumption. The Strömgren colours have only a weak sensitivity to surface gravity $(\sim \pm 15 \mathrm{~K}$ per \pm 0.1 dex in $\log g$ ), which is also true for neutral iron lines (the differential impact on the iron abundance inferred from lowand high-excitation lines is 0.001 dex per $0.1 \mathrm{dex}$ in $\log g$, which translates to $<0.5 \mathrm{~K}$ in effective temperature.). The influence of a higher helium-to-hydrogen ratio on the $\mathrm{H} \alpha$ profile has been investigated and found to be negligible as well, if the number density of metals remains constant. Based on the information in Table 2, we conclude that the difference in effective temperature between the stars is likely to be small. We assume that they have the same effective temperature and adopt $10 \mathrm{~K}$ as a representative error in the relative difference.

The photometric surface gravities were determined in Paper I, from the common relation between mass, surface gravity, luminosity, and temperature. Assuming $M=0.8 M_{\odot}$ and bolometric correction $\mathrm{BC}=-0.390$, the resulting values are different by 0.03 dex only. However, as the evolution of a heliumenhanced star is certainly different from that of a helium-normal one, the assumption of equal masses for the two stars may be wrong. If \#14565 is helium-enriched compared to \#5644, it should, from stellar-evolution arguments, have a lower mass and consequently a lower surface gravity. We also calculated surface gravities based on the ionisation equilibrium of Fe I and Fe II. With a normal-star composition for both objects, the surface gravity values are different by 0.02 dex. If a higher helium mass fraction is assumed for 14565 , the surface gravity value
Table 3. Iron mass fractions derived for \#5644 and \#14 565, assuming different mass fractions of helium.

\begin{tabular}{lllll}
\hline \hline $\mathrm{ID}$ & 5644 & 14565 & 14565 & 14565 \\
\hline$y$ & 0.085 & 0.085 & 0.170 & 1.000 \\
$Y$ & 0.253 & 0.253 & 0.405 & 0.800 \\
$X$ & 0.747 & 0.747 & 0.595 & 0.200 \\
$\log g$ & 1.50 & 1.52 & 1.45 & 1.21 \\
$A(\mathrm{Fe})$ & 5.316 & 5.323 & 5.323 & 5.323 \\
$Z(\mathrm{Fe}) \times 10^{6}$ & 8.662 & 8.803 & 7.007 & 2.353 \\
\hline
\end{tabular}

that establishes the equilibrium will shift to lower values in accordance with Eq. (1). However, the iron abundance found relative to hydrogen will be the same in both cases. We thus conclude that if the stars have the same helium mass fraction, they also have almost the same surface gravity. If not, the difference in $\log g$ cannot be determined independently from the difference in helium content. However, if $\log g$ is calculated by ionisation equilibrium, the effect of the helium enhancement will effectively cancel and $[\mathrm{Fe} / \mathrm{H}]$ will be found to be the same.

We calculate the iron abundances, $A(\mathrm{Fe})$, and iron mass fractions, $Z(\mathrm{Fe})$, of 5644 and 14565 , using $T_{\text {eff }}=4750 \mathrm{~K}$, $\xi_{t}=1.7 \mathrm{kms}^{-1}$, and the surface gravities that satisfy the ionisation equilibrium of iron, which is different for different values of $y$. The results are given in Table 3. When assuming a normal He composition, the iron abundances of the stars agree to within 0.007 dex. A higher mass fraction of helium in 14565 will not affect the iron abundances derived relative to hydrogen, but the mass fraction of iron will be lower. Assuming that the mass fractions of iron are exactly the same for the stars, we can calculate the corresponding difference in helium mass fraction. The errors in $\Delta A(\mathrm{Fe})$ and $\Delta Z(\mathrm{Fe})$ are estimated from the standard deviation in the mean difference for each individual line, to cancel contributions from uncertainties in oscillator strengths. The resulting error is 0.003 dex for Fe I and 0.009 dex for Fe II. Adopting a relative uncertainty in effective temperature of $10 \mathrm{~K}$ corresponds to an additional $0.016 \mathrm{dex}$. The total error budget is thus $0.028 \mathrm{dex}$, or $6.7 \%$ in iron mass fraction. Assuming that $\# 14565$ have the same $Z(\mathrm{Fe})$ as \#5644, $\Delta Y$ between the stars then becomes $0.01 \pm 0.06$. We caution that this is far from a stringent constraint on the true difference in $Y$, especially considering the possibility of a real (small) difference in iron mass fraction. However, it is safe to say that our simple arithmetic exercise clearly does not support extreme He-enhancement, in agreement with the photometric tightness of the main sequence.

In the cluster formation scenario described by Decressin et al. (2007a), in which slow winds from rotating massive stars pollute the star forming gas, one expects an anti-correlation between helium and $[\mathrm{O} / \mathrm{Na}]$ for second generation stars born out of a mixture of stellar ejecta and pristine material. As can be seen in Fig. 13 of Decressin et al., the small value implied for $\Delta Y$ is fully compatible with the corresponding $\Delta[\mathrm{O} / \mathrm{Na}]$ of 0.65 dex between \#5644 and \#14 565. This good agreement persists over the whole mass range considered by Decressin et al. (2007a), i.e., for polluter stars with masses between 20 and $120 M_{\odot}$.

\section{Consequences for the evolutionary scenario of NGC 6397}

We now investigate whether the abundance patterns we derived for NGC 6397 can be accounted for in the framework of the socalled "wind of fast rotating massive stars" (WFRMS) scenario (see e.g. Prantzos \& Charbonnel 2006; Decressin et al. 2007a,b). 
In this scenario, second generation stars are formed from matter ejected through slow winds of individual massive stars $(M \geq$ $20 M_{\odot}$ ), mixed locally with pristine interstellar gas.

We note from the onset that the main alternative scenario, proposing that ejecta from intermediate-mass AGB stars have shaped the abundances patterns of light elements may be equally successful in explaining the observed anti-correlation of $\mathrm{O}$ and $\mathrm{Na}$, as well as an accompanying mild He enrichment. In particular, Ventura \& D'Antona (2009) show how the O-Na data of Carretta et al. (2005) can be reproduced if 50\% of AGB ejecta are mixed with $50 \%$ pristine intracluster gas. The main argument against such a scenario is the implied near constancy of $\mathrm{C}+\mathrm{N}+\mathrm{O}$, as discussed above. Decressin et al. (2009) demonstrate how metal-poor massive rotating AGBs are expected to produce very strong $\mathrm{C}+\mathrm{N}+\mathrm{O}$ enhancement as He-burning products are also released in the ejecta. In contrast, for the massive star scenario only H-burning products are expected to enter the composition of second generation stars, thus maintaining a stable sum of $\mathrm{C}+\mathrm{N}+\mathrm{O}$.

\subsection{Method}

We follow the same procedure as described in Decressin et al. (2007a), using the stellar models of Decressin et al. (2007b) computed for the metallicity of NGC $6752([\mathrm{Fe} / \mathrm{H}] \sim-1.56)$, i.e. slightly higher than that of NGC $6397([\mathrm{Fe} / \mathrm{H}] \sim-2.08)$. A difference of this size should have no impact on our conclusions (Ekström et al. 2008). However, the enrichment history of the two clusters certainly differ to some extent, which must be considered in the analysis. In particular, the full range in $\mathrm{O}$ abundance is twice as large in NGC 6752 (0.8 dex, Yong et al. 2003) than in NGC 6397 (0.4 dex, see Fig. 6). In addition, NGC 6752 has a broadened main sequence (Milone et al. 2010) and an extended horizontal branch, indicative of a larger variation in the He content.

When establishing the recycling process of NGC6752, Decressin et al. (2007a) took into account all the material ejected by massive stars rotating at critical velocity, both on the main sequence and during the first part of the central He-burning phase ${ }^{2}$. This allowed the build-up of extreme abundance anomalies in both $\mathrm{O}$ and $\mathrm{Na}$, and also lead to high He-enrichment for the "extreme component" of second generation stars. As discussed in Sects. 3.1 and 3.5, such extreme anomalies appear to be lacking in NGC 6397, and the second generation stars are probably only mildly overabundant in He. Consequently, we assume that only the slow winds ejected on the main sequence by rapidly rotating massive stars are recycled into the second generation and we neglect the material that is lost during the luminous blue variable phase. As discussed in Sect. 4.5, this may have some impact on the total mass budget, although most of the mass loss through the slow mechanical winds occurs while the rapidly rotating stars are on the main sequence (in the case of a $60 M_{\odot}$ rotating at critical velocity, $20 M_{\odot}$ and $7 M_{\odot}$ are lost through the mechanical wind on and slightly after the main sequence, respectively, according to these models). We do not venture to discuss in detail the underlying reason for the different observed abundance patterns in NGC 6752 and NGC 6397, but one may speculate that different initial masses of these clusters have played a role, especially on

\footnotetext{
${ }^{2}$ Owing to strong mechanical mass loss the models predict that rapidly rotating stars actually evolve away from the critical limit during the central He-burning phase, before the He-burning products contaminate the slow wind component (see Decressin et al. 2007b; Ekström et al. 2008).
}

the amount of pristine gas available to be mixed with the massive star ejecta to form second generation stars (see Sect. 4.2). We note also that cluster mass has been identified as one of the main parameters governing the chemical patterns of GCs, together with cluster metallicity, age (see e.g. Carretta et al. 2009a, 2010), and environment of formation (Fraix-Burnet et al. 2009).

\subsection{Amount of dilution between massive star ejecta and pristine gas}

The star-to-star spread in light element abundances observed in globular clusters today may imply that all the material ejected by the first generation massive stars was not fully mixed before being recycled into the second stellar generation. In addition, the measurements of both $\mathrm{O}$ and $\mathrm{Li}$ pinpoints the formation of second generation stars in the vicinity of individual massive stars from their slow winds mixed with pristine interstellar matter.

As explained in Decressin et al. (2007a) we may use the observed $\mathrm{Li}$-Na anti-correlation to estimate the amount of dilution between the massive star ejecta that are totally void of $\mathrm{Li}$ and the pristine intracluster gas left after the formation of first generation stars, whose Li abundance is assumed to be equal to the primordial (Big Bang) value. In addition, we assume that the slow wind of each individual massive star experiences locally a variable dilution with time. In the early main-sequence phase, the stellar ejecta encounter more pristine gas and are hence more diluted than the matter ejected later. As the stellar winds become more Na-rich later in the course of the evolution of a massive polluter, the Li-Na anti-correlation builds up naturally: a second generation of stars of a relatively high Li content and a relatively low $\mathrm{Na}$ abundance are created first, while stars formed from matter ejected later have less $\mathrm{Li}$ and more $\mathrm{Na}$.

In Fig. 9, we present theoretical predictions for the $\mathrm{Li}$ and $\mathrm{Na}$ abundance variations in the resulting material out of which second generation low-mass stars are assumed to form. The tracks shown correspond to the cases of the ejecta of the $20 M_{\odot}$ and $120 M_{\odot}$ models by Decressin et al. (2007b) for different values $(0.2,0.4,0.6$, and 0.8$)$ of the minimum dilution (along individual tracks dilution decreases from 1.0 to this minimum value as the polluter star evolves). Predictions for polluters with different initial masses would fill the whole space between 20 and $120 M_{\odot}$ tracks. The initial (pristine) $[\mathrm{Na} / \mathrm{Fe}]$ value is assumed to be the average that we find for first generation stars (i.e., -0.3 ; see Sect. 3.1). For Li, we consider the value from WMAP and Big Bang nucleosynthesis $(A(\mathrm{Li})=2.72$ according to Cyburt et al. 2008) and take into account Li depletion of 0.2 dex at the surface of both first and second generation low-mass stars during the main sequence (see Charbonnel \& Primas 2005, and Paper I). In Fig. 9, the theoretical tracks are superimposed on the $\mathrm{Li}$ and $\mathrm{Na}$ observational data by Paper I for NGC 6397 TO and SGB stars that have not yet undergone the first dredge-up. TO and SGB stars (defined here as stars fainter and brighter than $V=16.2$, respectively) are indicated by different symbols to also consider the 0.1 dex difference in $A(\mathrm{Li})$ found between these two groups, which in Paper I was interpreted as a sign of a dredge-up of settled Li. Various degrees of dilution can accurately explain the observed spread, and the bulk of the sample can be reproduced assuming a minimum dilution factor of 0.5 (i.e., that at least $50 \%$ of the material that formed the corresponding stars is pristine gas). Stars with low-Li and high-Na stars require a lower minimum dilution factor of $\sim 0.2$. Within the observational errors, the overall agreement between the observational and theoretical trends and dispersions is satisfactory. 


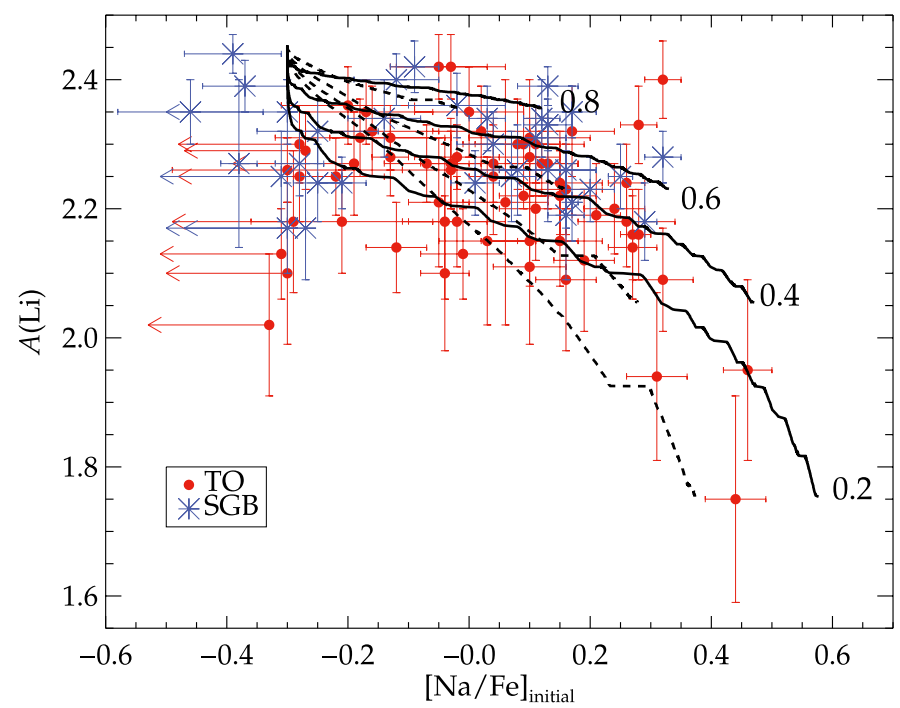

Fig. 9. $\mathrm{Li}$ and $\mathrm{Na}$ abundances for TO and SGB stars in NGC 6397 (data from Paper I, with improved non-LTE derivation). The initial $[\mathrm{Na} / \mathrm{Fe}]$, shown on the abscissa, has been estimated by correcting $A(\mathrm{Na})$ for atomic diffusion by +0.2 dex and assuming $[\mathrm{Fe} / \mathrm{H}]=-2.1$ for all stars. The red bullets represent turn-off stars and the blue stars subgiants. Superimposed are the theoretical predictions for the chemical composition of the matter out of which second generation low-mass stars are expected to form in the WFRMS scenario. The tracks shown correspond to the cases of $20 M_{\odot}$ and $120 M_{\odot}$ polluter stars (dashed and full lines, respectively) assuming different values $(0.2,0.4,0.6,0.8)$ for the minimum dilution coefficient. See text for more details.

\subsection{Expected anti-correlations}

Armed with this calibration for the dilution between massive star ejecta and pristine gas we can now make similar predictions for the (anti-)correlations between $\mathrm{O}, \mathrm{Na}$, and $\mathrm{Al}$. For the composition of the pristine cluster gas, we use the average values that we derived for first generation stars for $\mathrm{O}$ and $\mathrm{Na}([\mathrm{O} / \mathrm{Fe}]=0.7$, $[\mathrm{Na} / \mathrm{Fe}]=-0.3)$. Since we could only derive upper limits to the $\mathrm{Al}$ abundance of the first generation, we assume a value that is approximately compatible with the field at this metallicity, $[\mathrm{Al} / \mathrm{Fe}]=0.0$ (see Fig. 7 )

The theoretical tracks for the chemical composition of material out of which second generation stars can form are shown in Fig. 10. They correspond to the case of a minimum dilution equal to 0.2 (for these elements, the tracks for other minimal dilution values are superimposed on one another although to different extents) for polluters of various initial masses. As can be seen, the tracks do not vary much with polluter mass since the central temperature in massive stars is always high enough to burn protons through $\mathrm{CNO}-$ cycles and $\mathrm{Ne}-\mathrm{Na}$ and $\mathrm{Mg}-\mathrm{Al}$ chains. However, the efficiency of the rotationally-induced mixing increases with stellar mass, implying that more massive stars give rise to more extreme abundance variations. In particular, only the most massive stars can be held responsible for the highest $\mathrm{Al}$ and $\mathrm{Na}$ enrichment observed in NGC 6397. Satisfactory agreement is again achieved between data and predictions.

Decressin et al. (2007b) described the sensitivity of the model predictions to the nuclear reaction rates adopted in the computations. The stellar models used here were computed with their "set C" for the nuclear reaction rates (see Table 2 of Decressin et al. 2007b). In this case, the total magnesium abundance decreases by $\sim 0.1$ dex only. If we were to assume an enhancement of the ${ }^{24} \mathrm{Mg}(p, \gamma)$ reaction as done in "set D" of Decressin et al. (2007b), we would however predict a total

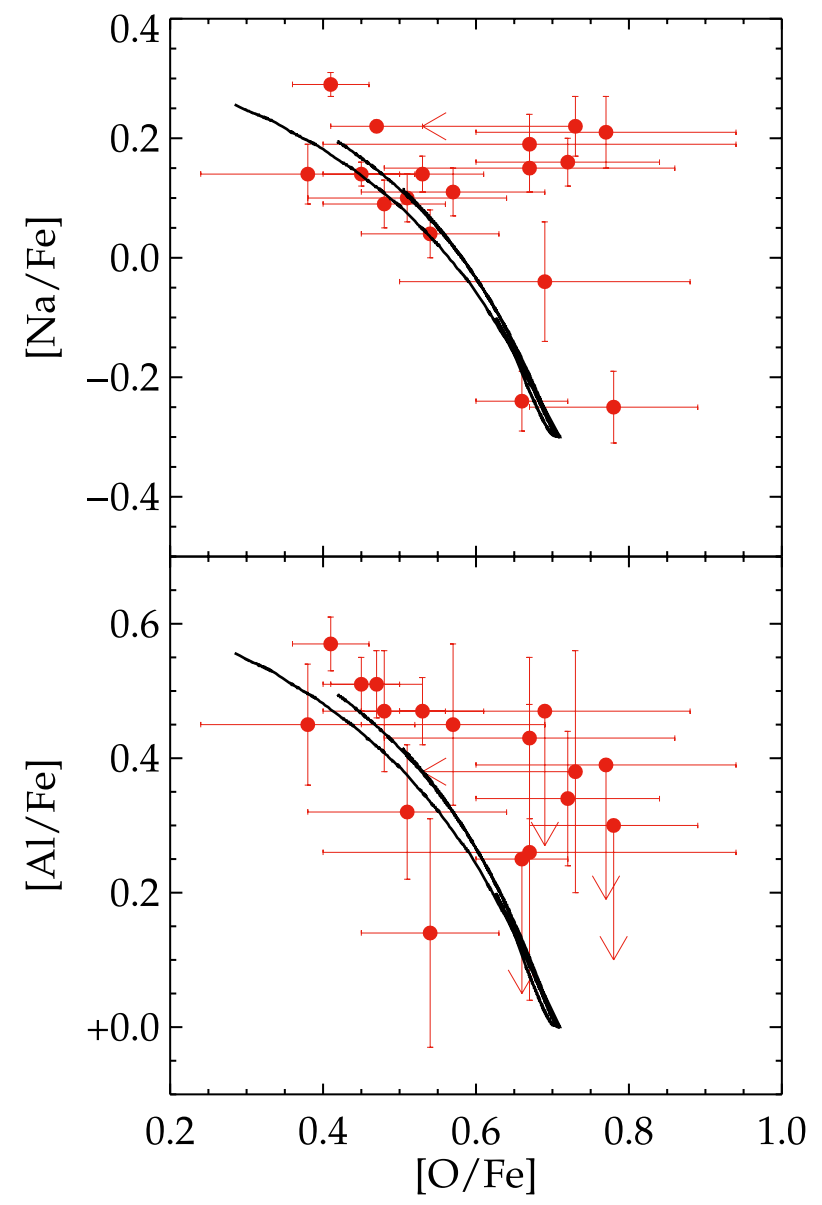

Fig. 10. O-Na (top) and O-Al (bottom) anti-correlations are shown for the giant stars of NGC 6397. Superimposed are the theoretical predictions for the chemical composition of the matter out of which second generation low-mass stars are expected to form. The tracks correspond to the cases of $20,40,60$, and $120 M_{\odot}$ polluter stars for a minimum value of 0.2 for the dilution coefficient.

magnesium abundance decrease by $\sim 0.15$ dex, in slightly better agreement with our Mg data in NGC 6397.

\subsection{He content}

We can now make predictions about the helium spread expected within the WFRMS scenario with the assumptions described above in the specific case of NGC 6397. Figure 11 shows the predicted variation in $\mathrm{He}$ mass fraction as a function of the $[\mathrm{O} / \mathrm{Na}]$ ratio. We also show the values estimated in Sect. 3.5 for \#5644 and \#14 565. We see that the theoretical helium variation corresponding to a $\Delta[\mathrm{O} / \mathrm{Na}]$ of 0.65 is smaller than 0.04 , fully compatible with the spectroscopic estimate, when very modest $\mathrm{C}+\mathrm{N}+\mathrm{O}$ enrichment is considered as discussed above.

When assuming a "standard" IMF (slope of 2.35) for the massive polluter stars and taking into account the variable dilution process described in Sect. 4.2, we find that $\sim 70 \%$ of second generation stars should be born with an helium mass fraction that is 0.02 higher than first generation stars. In addition, about $5-10 \%$ of second generation stars are expected to be born within the range $Y=0.35-0.39$. The resulting dispersion in $Y$ is compatible with the constraints obtained from the width of the main sequence $(\Delta Y<0.04)$, although no blue, very He-rich main sequence has been identified in the cluster. This prediction is, 


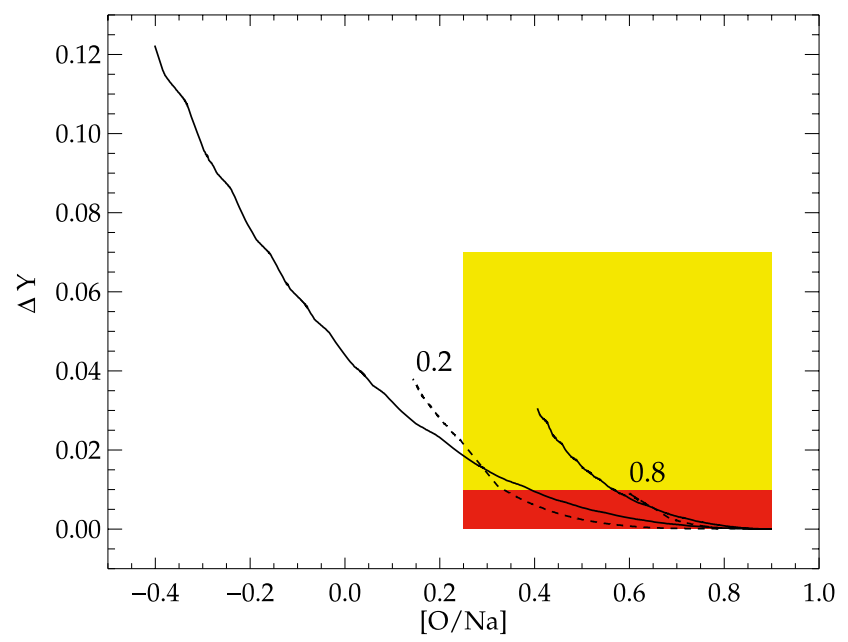

Fig. 11. Expected anti-correlation between helium (in mass fraction) and $[\mathrm{O} / \mathrm{Na}]$ for second generation stars (at birth) born out of material ejected through slow winds of massive stars and diluted with gas of pristine composition. The tracks correspond to the cases of 20 and $120 M_{\odot}$ polluter stars (dashed and full lines, respectively) assuming different values $(0.2$ and 0.8$)$ for the minimum dilution coefficient. See text for more details. The shaded regions indicate the star-to-star abundance differences derived in Sect. 3.5 for \#5644 and \#14 565 and related uncertainties $(\Delta[\mathrm{O} / \mathrm{Na}]=0.65$ and $\Delta Y<0.07)$.

however, at odds with the lack of a blue tail to the horizontal branch in NGC 6397.

\subsection{Initial cluster mass}

As discussed in Sect.3.1, about $75 \%$ of the long-lived lowmass stars still present today in NGC 6397 belong to the socalled second generation, while about $25 \%$ were born with the same chemical composition as the massive polluters. Prantzos \& Charbonnel (2006) and Decressin et al. (2007a,b) demonstrated that such high a number ratio of second generation stars requires either a very flat IMF for the first generation of massive stars, or that a very large number of first-generation low-mass stars have escaped the cluster.

We thus follow here the second scenario, assuming in addition that the first stellar generation contains stars with initial masses between 0.1 and $120 M_{\odot}$, while the second generation consists only of long-lived low-mass stars $\left(0.1-0.8 M_{\odot}\right)$. This assumption was introduced by Prantzos \& Charbonnel (2006) as a simplification and to maximise the ratio of ejecta to live stars. Simulations of star formation in the vicinity of rapidly rotating massive stars exhibiting disks are required to validate this hypothesis on physical grounds. We use a Salpeter IMF for the first generation of massive stars. On the other hand, we consider that the present-day log-normal distribution derived by Paresce $\&$ De Marchi (2000) reflects the IMF of the long-lived low-mass stars of both the first and second generation, thus neglecting the probable scenario that low-mass stars have been preferentially evaporated from the dynamically evolved cluster (see e.g. De Marchi et al. 2000).

We can then derive the amount of first-generation long-lived stars that must have been lost in order to fit the high ratio of second to first generation stars $3: 1$ ) found in NGC 6397. As we consider pollution by massive star ejecta released on the main sequence only, we have less polluter material to form new stars than in the case of NGC 6752, as studied by Decressin et al. (2007a). This effect is partially compensated by the higher dilution needed to reproduce the Li-Na anti-correlation as we form more second generation stars per unit mass released into slow winds.

Finally, we find that about $90 \%$ of first generation low-mass stars must have been lost by NGC 6397, probably during the early event of gas expulsion by supernovae (see Decressin et al. 2010). The initial mass of NGC 6397 must thus have been at least ten times higher than its present day mass. In this estimate, we neglect the long-term dynamical evolution processes that occur later on, when the two generations are dynamically mixed, i.e., have the same radial distribution, and should be ejected from the cluster at the same rate (Decressin et al. 2008). This late evolution can thus not be constrained by the present fraction of firstto-second generation stars.

\section{Conclusions}

By studying red giant branch stars in the globular cluster NGC 6397, we have demonstrated the possibility of distinguishing between the present stellar generations spectroscopically, by making use of a double-peaked histogram of $\mathrm{Na}$ abundances. A two-population fit returns a $\mathrm{Na}$ abundance similar to the halo field for $25 \%$ of the stars, which we characterise as belonging to a first generation, whereas the remaining $75 \%$ (i.e. second generation) have highly elevated $\mathrm{Na}$ abundances. This bimodal abundance signature should be verified for an extended sample, and similar histograms may also be possible to produce for $\mathrm{N}$ and $\mathrm{Al}$ abundances, for which large spreads are present in globular clusters. The abundance spreads are smaller for $\mathrm{O}$ and $\mathrm{Mg}$, making the task more challenging. Highly precise abundance analysis, with small statistical error bars will be necessary to identify these patterns, if indeed present.

Based on 17 different elements heavier than $\mathrm{Al}$, we conclude that there is no evidence that $\alpha$, iron-peak or neutron-capture abundances are significantly different between the stellar generations. We have also estimated the difference in He abundance between two stars of each generation by enforcing the mass fraction of iron to be same within the error bars of the analysis. This small (if any) difference in $\mathrm{He}$ is expected from models of cluster self-enrichment and also supported by the tightness of the main sequence of NGC 6397. The same exercise should be performed for other globular clusters, especially those displaying multiple main sequences, hopefully bringing us closer to identifying the process responsible for early cluster pollution.

We have finally shown that the abundance patterns observed in NGC 6397 can be well reproduced within the "wind of fast rotating massive stars" scenario. On the basis of the Li-Na relation we have been able to infer the dilution factors between the ejecta of massive polluters and interstellar gas of pristine composition. The observed ratio of second to first generation stars was used to estimate the number fraction of first generation long-lived lowmass stars that must have been lost by NGC 6397, and thus a lower limit to the initial mass of this globular cluster.

Acknowledgements. We thank M. Bergemann for helpful advice and E. Carretta for communicating data. The referee is thanked for many constructive comments to the submitted paper.

\section{References}

Andrievsky, S. M., Spite, M., Korotin, S. A., et al. 2007, A\&A, 464, 1081 Andrievsky, S. M., Spite, M., Korotin, S. A., et al. 2008, A\&A, 481, 481 Andrievsky, S. M., Spite, M., Korotin, S. A., et al. 2010, A\&A, 509, A88 Asplund, M. 2005, ARA\&A, 43, 481 
Asplund, M., Gustafsson, B., Kiselman, D., \& Eriksson, K. 1997, A\&A, 318, 521

Asplund, M., Grevesse, N., Sauval, A. J., \& Scott, P. 2009, ARA\&A, 47, 481

Barklem, P. S., Christlieb, N., Beers, T. C., et al. 2005, A\&A, 439, 129

Bell, R. A., Dickens, R. J., \& Gustafsson, B. 1979, ApJ, 229, 604

Bergemann, M., \& Gehren, T. 2008, A\&A, 492, 823

Bielski, A. 1975, J. Quant. Spectr. Radiat. Transf., 15, 463

Biemont, E., \& Zeippen, C. J. 1992, A\&A, 265, 850

Briley, M. M., Bell, R. A., Hoban, S., \& Dickens, R. J. 1990, ApJ, 359, 307

Burris, D. L., Pilachowski, C. A., Armandroff, T. E., et al. 2000, ApJ, 544, 302

Carretta, E., Gratton, R. G., Lucatello, S., Bragaglia, A., \& Bonifacio, P. 2005, A\&A, 433, 597

Carretta, E., Bragaglia, A., Gratton, R., \& Lucatello, S. 2009a, A\&A, 505, 139

Carretta, E., Bragaglia, A., Gratton, R. G., et al. 2009b, A\&A, 505, 117

Carretta, E., Bragaglia, A., Gratton, R. G., et al. 2010, A\&A, 516, A55

Castilho, B. V., Pasquini, L., Allen, D. M., Barbuy, B., \& Molaro, P. 2000, A\&A, 361,92

Cayrel, R., Depagne, E., Spite, M., et al. 2004, A\&A, 416, 1117

Chang, T. N. 1990, Phys. Rev. A, 41, 4922

Charbonnel, C. 2005, in From Lithium to Uranium: Elemental Tracers of Early Cosmic Evolution, ed. V. Hill, P. François, \& F. Primas, IAU Symp., 228, 347

Charbonnel, C. 2007, in Why Galaxies Care About AGB Stars: Their Importance as Actors and Probes, ed. F. Kerschbaum, C. Charbonnel, \& R. F. Wing, ASP Conf. Ser., 378, 416

Charbonnel, C., \& Lagarde, N. 2010, A\&A, 522, A10

Charbonnel, C., \& Primas, F. 2005, A\&A, 442, 961

Cyburt, R. H., Fields, B. D., \& Olive, K. A. 2008, J. Cosmol. Astro-Part. Phys., 11,12

D'Antona, F., \& Caloi, V. 2008, MNRAS, 390, 693

De Marchi, G., Paresce, F., \& Pulone, L. 2000, ApJ, 530, 342

de Mink, S. E., Pols, O. R., Langer, N., \& Izzard, R. G. 2009, A\&A, 507, L1

Decressin, T., Charbonnel, C., \& Meynet, G. 2007a, A\&A, 475, 859

Decressin, T., Meynet, G., Charbonnel, C., Prantzos, N., \& Ekström, S. 2007b, A\&A, 464, 1029

Decressin, T., Baumgardt, H., \& Kroupa, P. 2008, A\&A, 492, 101

Decressin, T., Charbonnel, C., Siess, L., et al. 2009, A\&A, 505, 727

Decressin, T., Baumgardt, H., Charbonnel, C., \& Kroupa, P. 2010, A\&A, 516, A73

Denisenkov, P. A., \& Denisenkova, S. N. 1989, Astronomicheskij Tsirkulyar, 1538,11

di Criscienzo, M., D’Antona, F., \& Ventura, P. 2010, A\&A, 511, A70

D’Orazi, V., Lucatello, S., Gratton, R., et al. 2010, ApJ, 713, L1

Ekström, S., Meynet, G., Maeder, A., \& Barblan, F. 2008, A\&A, 478, 467

Fabbian, D., Asplund, M., Barklem, P. S., Carlsson, M., \& Kiselman, D. 2009, A\&A, 500, 1221

Fraix-Burnet, D., Davoust, E., \& Charbonnel, C. 2009, MNRAS, 398, 1706

François, P., Depagne, E., Hill, V., et al. 2007, A\&A, 476, 935

Fuhr, J. R., Martin, G. A., \& Wiese, W. L. 1988, Atomic transition probabilities. Scandium through Manganese (New York: American Institute of Physics (AIP) and American Chemical Society)

Gehren, T., Shi, J. R., Zhang, H. W., Zhao, G., \& Korn, A. J. 2006, A\&A, 451, 1065

Gratton, R. G., Bonifacio, P., Bragaglia, A., et al. 2001, A\&A, 369, 87

Gratton, R., Sneden, C., \& Carretta, E. 2004, ARA\&A, 42, 385

Grundahl, F., Briley, M., Nissen, P. E., \& Feltzing, S. 2002, A\&A, 385, L14

Gustafsson, B., Edvardsson, B., Eriksson, K., et al. 2008, A\&A, 486, 951

Hannaford, P., Lowe, R. M., Grevesse, N., Biemont, E., \& Whaling, W. 1982, ApJ, 261, 736

James, G., François, P., Bonifacio, P., et al. 2004, A\&A, 427, 825

Kiselman, D. 2001, New Astron. Rev., 45, 559

Koch, A., \& McWilliam, A. 2008, AJ, 135, 1551

Komarovskii, V. A. 1991, Optics and Spectroscopy, 71, 322
Korn, A. J., Grundahl, F., Richard, O., et al. 2007, ApJ, 671, 402

Langer, G. E., Hoffman, R., \& Sneden, C. 1993, PASP, 105, 301

Lawler, J. E., \& Dakin, J. T. 1989, J. Opt. Soc. Am. B Opt. Phys., 6, 1457

Lawler, J. E., Wickliffe, M. E., den Hartog, E. A., \& Sneden, C. 2001, ApJ, 563, 1075

Lincke, R., \& Ziegenbein, B. 1971, Z. Physik, 241, 369

Lind, K., Korn, A. J., Barklem, P. S., \& Grundahl, F. 2008, A\&A, 490, 777

Lind, K., Primas, F., Charbonnel, C., Grundahl, F., \& Asplund, M. 2009, A\&A, 503,545

Lind, K., Asplund, M., Barklem, P. S., \& Belyaev, A. K. 2011, A\&A, to be published,

Ljung, G., Nilsson, H., Asplund, M., \& Johansson, S. 2006, A\&A, 456, 1181

Marino, A. F., Villanova, S., Piotto, G., et al. 2008, A\&A, 490, 625

Martin, G. A., Fuhr, J. R., \& Wiese, W. L. 1988, Phys. Chem. Ref. Data, 17, 3

Miles, B. M., \& Wiese, W. L. 1969, Atomic Data, 1, 1

Milone, A. P., Piotto, G., King, I. R., et al. 2010, ApJ, 709, 1183

Nissen, P. E., Primas, F., Asplund, M., \& Lambert, D. L. 2002, A\&A, 390, 235

Norris, J. E. 2004, ApJ, 612, L25

Norris, J. E., Ryan, S. G., \& Beers, T. C. 2001, ApJ, 561, 1034

Önehag, A., Gustafsson, B., Eriksson, K., \& Edvardsson, B. 2009, A\&A, 498, 527

Paresce, F., \& De Marchi, G. 2000, ApJ, 534, 870

Pasquini, L., Bonifacio, P., Randich, S., Galli, D., \& Gratton, R. G. 2004, A\&A, 426,651

Pasquini, L., Ecuvillon, A., Bonifacio, P., \& Wolff, B. 2008, A\&A, 489, 315

Pereira, T. M. D., Asplund, M., \& Kiselman, D. 2009, A\&A, 508, 1403

Pickering, J. C., Thorne, A. P., \& Perez, R. 2001, ApJS, 132, 403

Prantzos, N., \& Charbonnel, C. 2006, A\&A, 458, 135

Primas, F., \& Sobeck, J. 2008, in Evolution and Nucleosynthesis in AGB Stars, ed. R. Guandalini, S. Palmerini, \& M. Busso, AIP Conf. Ser., 1001, 230

Pumo, M. L., D’Antona, F., \& Ventura, P. 2008, ApJ, 672, L25

Richard, O., Michaud, G., \& Richer, J. 2005, ApJ, 619, 538

Richer, H. B., Dotter, A., Hurley, J., et al. 2008, AJ, 135, 2141

Shimanskaya, N. N., Mashonkina, L. I., \& Sakhibullin, N. A. 2000, Astron. Rep., 44,530

Smith, G. 1988, J. Phys. B Atom. Mol. Phys., 21, 2827

Smith, G., \& O'Neill, J. A. 1975, A\&A, 38, 1

Smith, G., \& Raggett, D. S. J. 1981, J. Phys. B Atom. Mol. Phys., 14, 4015

Storey, P. J., \& Zeippen, C. J. 2000, MNRAS, 312, 813

Strömgren, B., Gustafsson, B., \& Olsen, E. H. 1982, PASP, 94, 5

Thévenin, F., Charbonnel, C., de Freitas Pacheco, J. A., et al. 2001, A\&A, 373, 905

Ventura, P., \& D’Antona, F. 2008, A\&A, 479, 805

Ventura, P., \& D'Antona, F. 2009, A\&A, 499, 835

Ventura, P., \& D'Antona, F. 2010, MNRAS, 402, L72

Warner, B. 1968, MNRAS, 140, 53

Wickliffe, M. E., \& Lawler, J. E. 1997, ApJS, 110, 163

Wiese, W. L., Smith, M. W., \& Glennon, B. M. 1966, Atomic transition probabilities, Vol. 1: Hydrogen through Neon, A critical data compilation, ed. W. L. Wiese, M. W. Smith, \& B. M. Glennon (NSRDS-NBS 4, Washington, DC: US Department of Commerce, National Bureau of Standards)

Wiese, W. L., Smith, M. W., \& Miles, B. M. 1969, Atomic transition probabilities, Vol. 2: Sodium through Calcium. A critical data compilation, ed. W. L. Wiese, M. W. Smith, \& B. M. Miles (NSRDS-NBS, Washington, DC: US Department of Commerce, National Bureau of Standards)

Yong, D., Grundahl, F., Lambert, D. L., Nissen, P. E., \& Shetrone, M. D. 2003, A\&A, 402, 985

Yong, D., Grundahl, F., Nissen, P. E., Jensen, H. R., \& Lambert, D. L. 2005, A\&A, 438, 875

Yong, D., Grundahl, F., Johnson, J. A., \& Asplund, M. 2008a, ApJ, 684, 1159

Yong, D., Karakas, A. I., Lambert, D. L., Chieffi, A., \& Limongi, M. 2008b, ApJ, 689,1031

Pages 14 to 16 are available in the electronic edition of the journal at http: //wWw . aanda.org 


\section{Appendix A: Line list and abundance tables}

Table A.1. Line list with reference to the adopted oscillator strength.

\begin{tabular}{|c|c|c|c|c|}
\hline Ion & $\begin{array}{c}\text { Wavelength } \\
{[\AA]}\end{array}$ & $\begin{array}{c}\chi_{l} \\
{[\mathrm{eV}]}\end{array}$ & $\overline{\log (g f)}$ & Ref. \\
\hline $\mathrm{OI}$ & 6300.304 & 0.000 & -9.819 & . \\
\hline O I & 7771.941 & 9.146 & 0.369 & 20 \\
\hline O I & 7774.161 & 9.146 & 0.223 & 20 \\
\hline $\mathrm{NaI}$ & 5682.633 & 2.102 & -0.706 & 2 \\
\hline $\mathrm{NaI}$ & 5688.194 & 2.104 & -1.406 & 2 \\
\hline $\mathrm{NaI}$ & 5688.205 & 2.104 & -0.452 & 2 \\
\hline $\mathrm{NaI}$ & 8183.256 & 2.102 & 0.237 & 2 \\
\hline $\mathrm{NaI}$ & 8194.791 & 2.104 & -0.462 & 2 \\
\hline $\mathrm{NaI}$ & 8194.824 & 2.104 & 0.492 & 2 \\
\hline Mg I & 5711.088 & 4.346 & -1.833 & 3 \\
\hline $\mathrm{Al} \mathrm{I}$ & 6696.023 & 3.143 & -1.347 & 4 \\
\hline Al I & 6696.185 & 4.022 & -1.576 & 5 \\
\hline Al I & 6698.673 & 3.143 & -1.647 & 4 \\
\hline Si I & 5665.555 & 4.920 & -1.750 & 5 \\
\hline Si I & 5690.425 & 4.930 & -1.769 & 5 \\
\hline Si I & 6155.134 & 5.619 & -0.754 & 5 \\
\hline Si I & 6237.319 & 5.614 & -0.975 & 5 \\
\hline $\mathrm{Ca} \mathrm{I}$ & 5349.465 & 2.709 & -0.310 & 6,7 \\
\hline $\mathrm{Ca} \mathrm{I}$ & 5512.980 & 2.933 & -0.464 & 7 \\
\hline $\mathrm{Ca} \mathrm{I}$ & 5581.965 & 2.523 & -0.555 & 6,7 \\
\hline $\mathrm{Ca} \mathrm{I}$ & 5588.749 & 2.526 & 0.358 & 6,7 \\
\hline $\mathrm{CaI}$ & 5590.114 & 2.521 & -0.571 & 6,7 \\
\hline $\mathrm{CaI}$ & 5601.277 & 2.526 & -0.523 & 6,7 \\
\hline $\mathrm{Ca} \mathrm{I}$ & 5857.451 & 2.933 & 0.240 & 7 \\
\hline $\mathrm{CaI}$ & 6102.723 & 1.879 & -0.793 & 8 \\
\hline $\mathrm{Ca} \mathrm{I}$ & 6122.217 & 1.886 & -0.316 & 8 \\
\hline $\mathrm{Ca} \mathrm{I}$ & 6162.173 & 1.899 & -0.090 & 8 \\
\hline $\mathrm{Ca} \mathrm{I}$ & 6166.439 & 2.521 & -1.142 & 6,7 \\
\hline $\mathrm{Ca} \mathrm{I}$ & 6439.075 & 2.526 & 0.390 & 6,7 \\
\hline $\mathrm{Ca} \mathrm{I}$ & 6449.808 & 2.521 & -0.502 & 6,7 \\
\hline $\mathrm{CaI}$ & 6471.662 & 2.526 & -0.686 & 6,7 \\
\hline $\mathrm{Ca} \mathrm{I}$ & 6493.781 & 2.521 & -0.109 & 6,7 \\
\hline $\mathrm{Ca} \mathrm{I}$ & 6499.650 & 2.523 & -0.818 & 6,7 \\
\hline $\mathrm{Sc}$ II & 5239.813 & 1.455 & -0.765 & 9 \\
\hline Sc II & 5526.790 & 1.768 & 0.024 & 9 \\
\hline $\mathrm{Sc}$ II & 5641.001 & 1.500 & -1.131 & 9 \\
\hline Sc II & 5669.042 & 1.500 & -1.200 & 9 \\
\hline $\mathrm{Sc}$ II & 5684.202 & 1.507 & -1.074 & 9 \\
\hline $\mathrm{Sc}$ II & 6245.637 & 1.507 & -1.030 & 5 \\
\hline Ti I & 4981.731 & 0.848 & 0.504 & 10 \\
\hline Ti I & 4999.503 & 0.826 & 0.250 & 10 \\
\hline Ti I & 5009.645 & 0.021 & -2.259 & 10 \\
\hline Ti I & 5016.161 & 0.848 & -0.574 & 10 \\
\hline Ti I & 5020.026 & 0.836 & -0.414 & 10 \\
\hline Ti I & 5025.570 & 2.041 & 0.250 & 10 \\
\hline Ti I & 5147.478 & 0.000 & -2.012 & 10 \\
\hline Ti I & 5210.385 & 0.048 & -0.884 & 10 \\
\hline Ti I & 5866.451 & 1.067 & -0.840 & 10 \\
\hline Ti I & 6258.102 & 1.443 & -0.355 & 10 \\
\hline Ti I & 6258.706 & 1.460 & -0.240 & 10 \\
\hline Ti II & 4779.985 & 2.048 & -1.260 & 5 \\
\hline Ti II & 4805.085 & 2.061 & -0.960 & 5 \\
\hline Ti II & 5005.157 & 1.566 & -2.720 & 11 \\
\hline Ti II & 5013.686 & 1.582 & -2.190 & 11 \\
\hline
\end{tabular}

Page 14 of 16 
Table A.1. continued.

\begin{tabular}{lcccc}
\hline \hline Ion & $\begin{array}{c}\text { Wavelength } \\
{[\AA]}\end{array}$ & $\begin{array}{c}\chi_{l} \\
{[\mathrm{eV}]}\end{array}$ & $\log (g f)$ & Ref. \\
\hline Ti II & 5418.768 & 1.582 & -2.000 & 11 \\
Cr I & 4922.265 & 3.104 & 0.270 & 10 \\
Cr I & 5329.138 & 2.914 & -0.064 & 10 \\
Cr II & 5237.329 & 4.073 & -1.160 & 10 \\
Cr II & 5313.563 & 4.074 & -1.650 & 10 \\
Mn I & 4783.427 & 2.298 & 0.042 & 10 \\
Co I & 5369.590 & 1.740 & -1.650 & 12 \\
Ni I & 4904.407 & 3.542 & -0.170 & 12 \\
Ni I & 4935.831 & 3.941 & -0.350 & 12 \\
Ni I & 4953.200 & 3.740 & -0.580 & 13 \\
Ni I & 4980.166 & 3.606 & 0.070 & 13 \\
Ni I & 5017.568 & 3.539 & -0.020 & 13 \\
Ni I & 5035.357 & 3.635 & 0.290 & 13 \\
Ni I & 5081.107 & 3.847 & 0.300 & 12 \\
Ni I & 5082.339 & 3.658 & -0.540 & 12 \\
Ni I & 5084.089 & 3.679 & 0.030 & 12 \\
Ni I & 5099.927 & 3.679 & -0.100 & 12 \\
Ni I & 5115.389 & 3.834 & -0.110 & 12 \\
Ni I & 5155.762 & 3.898 & 0.011 & 5 \\
Ni I & 6176.807 & 4.088 & -0.260 & 13 \\
Cu I & 5105.537 & 1.389 & -1.516 & 14 \\
Zn I & 4810.528 & 4.078 & -0.137 & 15 \\
Y II & 5087.416 & 1.084 & -0.170 & 16 \\
Y II & 5200.406 & 0.992 & -0.570 & 16 \\
Zr II & 5112.270 & 1.665 & -0.850 & 17 \\
Ba II & 5853.668 & 0.604 & -1.000 & 18 \\
Ba II & 6496.897 & 0.604 & -0.377 & 18 \\
Ce II & 5274.229 & 1.044 & -0.320 & 21 \\
Nd II & 4959.119 & 0.064 & -0.800 & 19 \\
Nd II & 5092.794 & 0.380 & -0.610 & 19 \\
Nd II & 5249.576 & 0.976 & 0.200 & 19 \\
Nd II & 5293.163 & 0.823 & 0.100 & 19 \\
Nd II & 5319.815 & 0.550 & -0.140 & 19 \\
Eu II & 6645.064 & 1.380 & 0.200 & 22 \\
\hline & & & & \\
\hline
\end{tabular}

References. ${ }^{1}$ Wiese et al. (1966); ${ }^{2}$ C. Froese Fischer (NIST); ${ }^{3}$ Lincke \& Ziegenbein (1971); ${ }^{4}$ Wiese et al. (1969); ${ }^{5}$ Vienna Atomic Line; ${ }^{6}$ Smith \& Raggett (1981); ${ }^{7}$ Smith (1988); ${ }^{8}$ Smith \& O'Neill (1975); ${ }^{9}$ Lawler \& Dakin (1989); ${ }^{10}$ Martin et al. (1988); ${ }^{11}$ Pickering et al. (2001); ${ }^{12}$ Fuhr et al. (1988); ${ }^{13}$ Wickliffe \& Lawler (1997); ${ }^{14}$ Bielski (1975); ${ }^{15}$ Warner (1968); ${ }^{16}$ Hannaford et al. (1982); ${ }^{17}$ Ljung et al. (2006); ${ }^{18}$ Miles \& Wiese (1969); ${ }^{19}$ Komarovskii (1991); ${ }^{20}$ Biemont \& Zeippen (1992); ${ }^{21}$ See ref. in Yong et al. (2005); ${ }^{22}$ Lawler et al. (2001). 


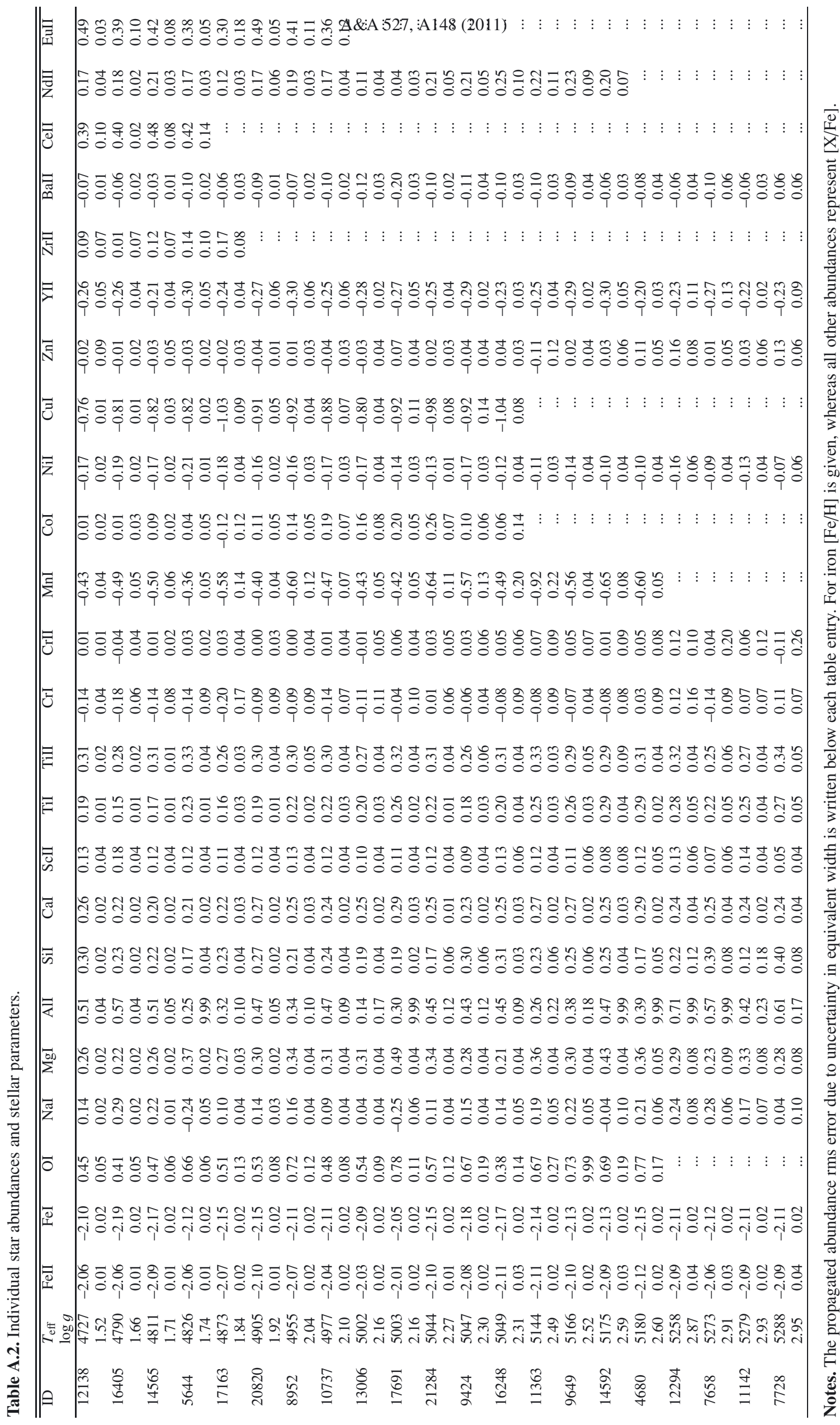

Page 16 of 16 